\title{
A Novel Two-Level Nested STAP Strategy for Clutter Suppression in Airborne Radar
}

\author{
Wei Wang $\mathbb{D}^{1,2}{ }^{1,2}$ Lin Zou, ${ }^{1}$ and Xuegang Wang ${ }^{1}$ \\ ${ }^{1}$ School of Information and Communication Engineering, University of Electronic Science and Technology of China, \\ Chengdu 611731, China \\ ${ }^{2}$ China Aerodynamics Research and Development Center, Mianyang 621000, China
}

Correspondence should be addressed to Wei Wang; 415382448@qq.com

Received 11 February 2019; Revised 14 May 2019; Accepted 23 May 2019; Published 4 June 2019

Academic Editor: Kishin Sadarangani

Copyright (C) 2019 Wei Wang et al. This is an open access article distributed under the Creative Commons Attribution License, which permits unrestricted use, distribution, and reproduction in any medium, provided the original work is properly cited.

\begin{abstract}
Nested arrays have been studied recently in array signal processing field because of their closed-form expressions for the sensor locations and achievable degrees of freedom (DOFs). In this paper, the concept of nesting is further extended to space-time adaptive processing (STAP). Different from the traditional uniform-STAP method that calculates the clutter plus noise covariance matrix (CNCM) and performs the STAP filter direct using the data snapshots collected from the uniform linear array (ULA) and the transmitting pulses with uniform pulse repetition interval (PRI), we present a new optimum two-level nested STAP (O2LN-STAP) strategy which employs an optimum two-level nested array (O2LNA) and an optimum two-level nested PRI (O2LN-PRI) to exploit the enhanced DOFs embedded in the space-time O2LN structure. Similar to the difference coarray perspective, we first construct a virtual space-time snapshot from the direct covariance matrix of the received signals. Then, a new CNCM estimation corresponding to the virtual space-time snapshot can be computed by the spatial-temporal smoothing technique for STAP filter. Furthermore, the comparative simulations and analyses with the traditional uniform-STAP and the recently reported coprime-STAP are carried out to verify the effectiveness of the O2LN-STAP approach.
\end{abstract}

\section{Introduction}

Space-time adaptive processing (STAP) is an attractive technique for clutter suppression and target detection in the airborne/spaceborne moving target indicator (MTI) radar [1-3]. It is well known that the STAP performance is commonly relevant to the effective system degrees of freedom (DOFs). For example, in contrast with the phased array (PA) STAP radar composed of uniform linear array (ULA), multiple-input-multiple-output (MIMO) STAP radar is more potential to detect a slow-moving weak target since the MIMO radar can gain larger DOFs due to its orthogonal transmitting waveforms [4]. However, these orthogonal waveforms are generated by a number of extra waveform control modules at the expense of design and control complexities.

Considering the fact that the PA radar is widely used in STAP applications, it is still valuable to find some valid ways to increase its effective DOFs. Intuitively, the DOFs of the
PA radar with ULA and uniform pulse repetition interval (PRI) can be simply increased by adding array elements and transmitting pulses. However, there may exist some issues for this uniform configuration. Firstly, the angle-Doppler ambiguities are unavoidable. Secondly, the electronic countercountermeasures (ECCM) capabilities are restricted by the uniform PRI [5]. Finally, it may be restricted by the large number of microwave modules, analog-to-digital converter (ADC) modules, and so on for the airborne radar applications where the significant constraints of equipment weight, size, hardware complexity, and power consumption are required [6]. On the other hand, the PA radar with nonuniform sparse (or thinned) array and PRI can provide an alternative way to heighten the DOFs while overcoming these issues [5-9]. Nevertheless, the methods with nonuniform sparse PRI require sparse recovery (SR) technique that has high computational complexity $[5,8]$. The STAP method in [7] does not use the SR technique, but it cannot avoid the typically high-average angle-Doppler side-lobe levels caused 
by randomly spaced sparse array and PRI [6, 9], which deteriorates the STAP performance of clutter suppression.

It is noted that the aforementioned approaches are all based on the physical nonuniform sparse antenna arrays. Actually, the virtual difference coarray concept with DOFs superiority has also been introduced into the nonuniform array signal processing domain. In the past years, this concept, which is able to reduce the average angle-Doppler side-lobe levels of the nonuniform sparse array without the usage of SR technique, has been applied to the directionof-arrival (DOA) estimation and the spatial beamforming. According to the array geometry, we classify those methods for exploiting the enhanced DOFs by use of the difference coarray perspective into three categories. The first one is based on the minimum redundancy array (MRA) [10]. It is shown that the DOFs of the MRA can be improved by constructing an augmented covariance matrix which is not positive semidefinite for finite number of snapshots [11, 12]. And in $[13,14]$, a transformation of this augmented matrix into a suitable positive definite Toeplitz matrix is suggested and an elaborate algorithm is presented to construct this Toeplitz matrix. Nevertheless, this class of approaches has two issues. One is that, for MRA, there are no closed-form expressions for the array geometry and the achievable DOFs for a given number of sensors, and the optimum design of such arrays is always complicated $[15,16]$. Another issue is that the algorithm for searching an appropriate augmented covariance matrix for a large array is a complex iterative procedure, which converges only to a local optimum $[13,14]$. Moreover, the second class of methods about the difference coarray is related to the recently developed coprime arrays, which have received much attention since they can be formulated more easily than the MRA while resolving more sources than the number of sensors $[17,18]$. In [19], performances of a generalized coprime array have been evaluated by use of their difference coarray equivalence, and this generalized coprime array has been applied to DOA estimation with both subspace-based method and sparse reconstruction technique. A new approach for passive beamforming and target detection using coprime array is also presented in [20], which can get the developments of peak side-lobe ratio and the integrated side-lobe ratio without giving rise to "ghost" targets in the presence of multiple interferers. Another multifrequency operation with coprime array is presented in [21] for increasing the number of resolvable sources in high-resolution DOA estimation. The authors employ multiple frequencies to fill the missing coarray elements, thereby enabling the coprime array to utilize all of the offered DOFs effectively. Finally, the techniques for further exploiting the increased DOFs using nested arrays $[22,23]$ can be regarded as the third class of difference coarray methods. An effective nested array design scheme can yield a fourth-order difference coarray to provide a higher number of DOFs for DOA estimation [24]. Another application for DOA estimation of the distributed sources with nested arrays has been studied in [25]. To use the nested array for wideband case, authors in [26] have proposed an effective strategy to apply the processing algorithm of nested array to each frequency component, and the whole spectral information of various frequencies has been cooperated to conduct the detection and estimation. Nested array has also been extended to MIMO radar for DOA estimation [27, 28].

In recent years, the difference coarray perspective has been considered as an effective approach to increase the system DOFs for STAP applications. To acquire more DOFs, authors in [29] have arranged the array geometry and the temporal sample interval to make the location of joint space-time samples satisfy minimum redundancy interval. However, this STAP algorithm is limited to a time-consuming procedure of computer searching. Moreover, a new STAP strategy for the airborne radar with space-time coprime sampling structure (which is referred to as coprime-STAP in the following) has been discussed in [30-32]. It is demonstrated that, using few array elements and transmitting pulses, the coprime-STAP method can achieve comparable performance with respect to the case of large ULA and great many pulses with uniform PRI. Additionally, a fully STAP with nested array is also presented in [33], where a high resolution in angle-Doppler plane is obtained. However, to complete this full STAP, the spatial and Doppler frequencies of all jamming and clutter sources need to be priorly known.

Inspired by the nesting concept in the spatial dimension, we think that using PRI with nested time interval might also obtain an increase of DOFs in the temporal (or Doppler) dimension. Therefore, following the difference coarray perspective, we have applied an optimum two-level nested STAP (O2LN-STAP) strategy to improve the STAP performance in this paper. Different from the traditional uniform-STAP method that calculates the clutter plus noise covariance matrix (CNCM) and performs the STAP filter direct using the received data collected from the physical ULA with uniform PRI, the STAP strategy shown in this paper presents a novel processing idea with an optimum two-level nested array (O2LNA) and an optimum twolevel nested PRI (O2LN-PRI). In our proposed method, we first construct a virtual space-time snapshot from the direct covariance matrix of the received signals. The virtual snapshot can be seen as the collection of the returned signals from a virtual large ULA and a large number of transmitting pulses with uniform PRI. Then, using the spatial-temporal smoothing approach [22], a new CNCM estimation of the virtual space-time snapshot can be computed for STAP filter. Remarkably, the existing coprime-STAP method, which is also considered in the virtual difference coarray domain, can get more DOFs than the traditional uniform-STAP that is implemented on the physical array and pulses. However, simulation results and comparative analyses in the following have indicated that the O2LN-STAP method can obtain more DOFs than both the traditional uniform-STAP and the coprime-STAP under the constraint of the same number of physical array elements and transmitting pulses, which makes a higher angle-Doppler resolution, lower side-lobe levels, larger signal-to-interference-plus-noise ratio (SINR), and better minimum detectable velocity (MDV) performances. On the other hand, for a specified number of DOFs and SINR level, the O2LN-STAP method can reach them with fewer physical array elements and transmitting pulses (i.e., less hardware complexity and power consumption) in contrast with the traditional uniform-STAP and the coprime-STAP. 


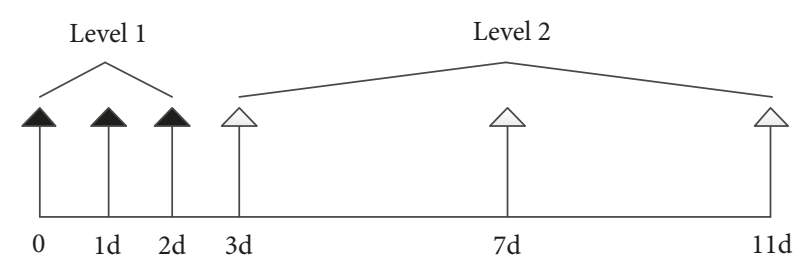

(a)

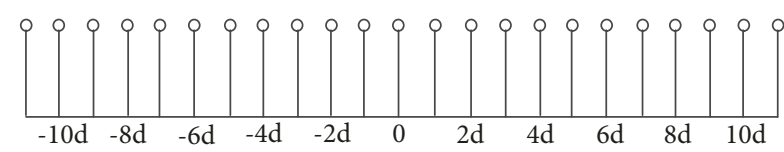

(b)

FIGURE 1: A two-level nested array and its difference coarray. (a) A two-level nested array with 3 sensors in each level. (b) The corresponding difference coarray.

The remainder of this paper is organized as follows. Section 2 introduces the nesting concept of array and PRI. The formulation of the O2LN-STAP strategy is deduced in Section 3. Section 4 gives simulation results and discussions to verify the effectiveness of the O2LN-STAP method. Finally, conclusion is presented in Section 5.

\section{Nesting Concept of Array and PRI}

2.1. Nested Array. We first briefly describe the nested array geometry in this subsection, and more details about it can be found in [22]. The nested array geometry can be generated very easily in a systematic fashion and we can exactly predict the DOFs of its difference coarray for a given number of sensors. Moreover, using this nested structure, it is indeed possible to generate $O\left(N^{2}\right)$ DOFs from $O(N)$ physical elements. The basic nesting strategy can generate a two-level nested array (2LNA) whose difference coarray is a filled ULA [22]. Though the idea of nesting is easy to be extended to higher dimensions, the nested arrays beyond two levels fail to produce an ULA difference coarray only using a second-order statistics of the received data [22]. Of course, it is also possible to produce a virtual filled ULA if we consider a higher-order statistics (e.g., the fourth-order cumulant) for the nested arrays beyond two levels [24]. However, using higher-order statistics usually brings hardly acceptable computational burden, which is not appropriate for practical applications. Considering that so many conventional array processing methods (such as beamforming algorithms) are based on the ULA, we prefer to deal with those signal processing issues in the ULA domain for simplicity and feasibility. Therefore, we just focus on the 2LNA in this paper since it can build a filled ULA with lower cost.

The 2LNA is basically a combination of two ULAs. One is called the inner ULA which has $N_{1}$ elements with spacing $d_{\text {in }}$. Another is named the outer ULA which has $N_{2}$ elements with spacing $d_{\text {out }}$ such that $d_{\text {out }}=\left(N_{1}+1\right) d_{\text {in }}$. In other words, it is a linear array with sensor locations given by the union of sets

$$
S_{i n}=\left\{n_{i n} d_{i n}, n_{\text {in }}=0,1, \ldots, N_{1}-1\right\}
$$

and

$$
S_{\text {out }}=\left\{n_{\text {out }}\left(N_{1}+1\right) d_{\text {in }}-d_{\text {in }}, n_{\text {out }}=1, \ldots, N_{2}\right\} .
$$

Obviously, the total number of 2LNA is $N=N_{1}+N_{2}$. Figure 1(a) illustrates a 2LNA with $N_{1}=N_{2}=3$ and $d=d_{i n}$, which is similar to the union of the transmitting and the receiving arrays of the MIMO radar and uses the same number of sensors.

Next, let us recall the definition of difference coarray [22]. Considering an array of $N$ sensors, with $\mathbf{p}_{i}$ denoting the position vector of the $i$ th sensor, a set is defined as

$$
D=\left\{\mathbf{p}_{i}-\mathbf{p}_{i^{\prime}}\right\}, \quad i=1,2, \ldots, N ; i^{\prime}=1,2, \ldots, N .
$$

We also define a set $D_{u}$ which consists of the distinct elements of the set $D$. Then, the difference coarray of a given physical array can be defined as a virtual array which has sensors located at the positions given by the set $D_{u}$. The difference coarray of the 2LNA presented in Figure 1(a) is shown in Figure 1(b). Note that the difference coarray of a 2LNA is a filled ULA with $\widehat{N}=2 N_{2}\left(N_{1}+1\right)-1$ elements whose positions are provided by the set

$$
\begin{aligned}
S_{c o} & =\left\{n_{c o} d_{i n}, n_{c o}=-N_{c o}, \ldots, N_{c o}, N_{c o}=N_{2}\left(N_{1}+1\right)\right. \\
& -1\}
\end{aligned}
$$

where $n_{c o}$ is an integer. It means that we can get $2 N_{2}\left(N_{1}+1\right)-1$ virtual elements (i.e., DOFs) in the coarray domain using only $N_{1}+N_{2}$ physical elements. In order to maximize the total DOFs of the coarray, authors in [22] have offered the optimal solutions of $N_{1}$ and $N_{2}$ for the expression $\widehat{N}=2 N_{2}\left(N_{1}+1\right)-1$ under the constraint of fixed total number of array sensors (i.e., $N=N_{1}+N_{2}$ is fixed), which can be given as follows.

Proposition 1. When $N$ is an even integer, the optimal solutions are $N_{1}=N_{2}=N / 2$; the corresponding maximum value of $\widehat{N}$ (i.e., DOFs) is $\left(N^{2}-2\right) / 2+N$.

Proposition 2. When $N$ is an odd integer, the optimal solutions are $N_{1}=(N-1) / 2$ and $N_{2}=(N+1) / 2$; the corresponding maximum value of $\widehat{N}$ (i.e., DOFs) is $\left(N^{2}-1\right) / 2+N$.

In the following contexts, we call the two-level nested array that satisfies Proposition 1 or Proposition 2 as the optimum two-level nested array (O2LNA).

2.2. Nested PRI. The nesting concept in array design can also be applied to PRI design for exploiting the enhanced DOFs in temporal (Doppler) dimension. Generally speaking, just using the parameter of time interval to replace the parameter of array element spacing defined in the nested array, we can still determine and analyze the property of the nested PRI. 


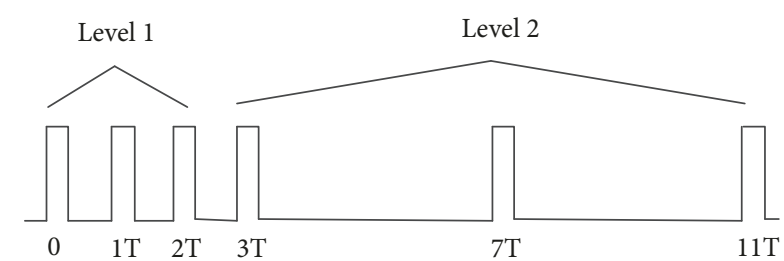

(a)

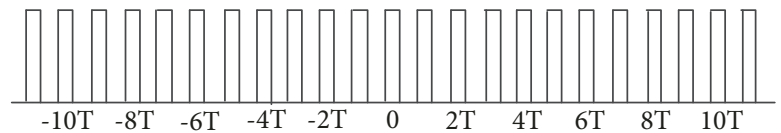

(b)

FIGURE 2: Transmitting pulses in a CPI with two-level nested PRI and its difference copulses. (a) A two-level nested PRI with 3 pulses in each level. (b) The corresponding difference copulses.

Specifically, we just describe the two-level nested PRI (2LN$\mathrm{PRI})$ in short due to the space limitation of this article. We assume that $M=M_{1}+M_{2}$ transmitting pulses with $2 \mathrm{LN}$ PRI are received in a coherent processing interval (CPI) for each range gate, where $M_{1}$ and $M_{2}$ have analogous meanings with $N_{1}$ and $N_{2}$, respectively. Like the construction of the 2LNA, the 2LN-PRI is also a combination of two uniform PRIs with constant time intervals $T_{\text {in }}$ and $T_{\text {out }}=\left(M_{1}+1\right) T_{\text {in }}$, respectively. Then, the pulse position can be given by the union of sets

$$
Q_{\text {in }}=\left\{m_{\text {in }} T_{\text {in }}, m_{\text {in }}=0,1, \ldots, M_{1}-1\right\}
$$

and

$$
Q_{\text {out }}=\left\{m_{\text {out }}\left(M_{1}+1\right) T_{\text {in }}-T_{\text {in }}, m_{\text {out }}=1, \ldots, M_{2}\right\} .
$$

Figure 2(a) presents an example of $2 \mathrm{LN}-\mathrm{PRI}$ in a CPI with $M_{1}=M_{2}=3$ and $T=T_{\text {in }}$.

Similar to the definition of difference coarray, we give a definition of difference copulses herein. Considering a CPI with $M$ pulses, using $\mathbf{q}_{j}$ denoting the timing position of the $j$ th pulse, a set is defined as

$$
G=\left\{\mathbf{q}_{j}-\mathbf{q}_{j^{\prime}}\right\}, \quad j=1,2, \ldots, M ; j^{\prime}=1,2, \ldots, M .
$$

Also defining a set $G_{u}$ which consists of the distinct elements of the set $G$, the difference copulses of the physical pulses in a CPI can be defined as a number of virtual pulses whose timing positions are given by the set $G_{u}$. For instance, Figure 2(b) shows the difference copulses corresponding to Figure 2(a). Note that the difference copulses of a 2LN-PRI in a CPI have $\widehat{M}=2 M_{2}\left(M_{1}+1\right)-1$ pulses with uniform PRI. The positions of those copulses are provided by the set

$$
\begin{aligned}
Q_{c o} & =\left\{m_{c o} T_{i n}, m_{c o}=-M_{c o}, \ldots, M_{c o}, M_{c o}\right. \\
& \left.=M_{2}\left(M_{1}+1\right)-1\right\}
\end{aligned}
$$

where $m_{c o}$ is an integer. It means that we can get $2 M_{2}\left(M_{1}+\right.$ 1) -1 virtual pulses (i.e., DOFs) in the copulses domain using only $M_{1}+M_{2}$ physical pulses. Furthermore, substituting $M_{1}$, $M_{2}$, and $M$ for $N_{1}, N_{2}$, and $N$ in Propositions 1 and 2, we can also construct an optimum two-level nested PRI (O2LNPRI), which will not be rewritten here for the limited length.

\section{Two-Level Nested STAP Strategy}

In this section, we further investigate the STAP with nested array and nested PRI to exploit the increasing of DOFs for improving STAP performance. We first present the STAP signal model with 2LNA and 2LN-PRI in Section 3.1. And then, in view of difference coarray and copulses perspectives, the strategy for virtual space-time snapshot construction is described in Section 3.2. Next, we employ the spatialtemporal smoothing technique to estimate the virtual CNCM matching to the virtual space-time snapshot in Section 3.3. At last, STAP filter based on the virtual snapshot and the virtual CNCM estimation is illustrated in Section 3.4.

3.1. STAP Signal Model with $2 L N A$ and $2 L N-P R I$. Consider a side-looking radar that consists of a 2LNA with $N=$ $N_{1}+N_{2}$ identical antenna elements whose locations can be determined by the union of sets $S_{\text {in }}$ and $S_{\text {out }}$. But herein, denoting $\lambda$ as the radar wavelength, we can rewrite the union as a new set $S=\left\{n d, n=0, n_{1}, n_{2}, \ldots, n_{N-1}\right\}$, where $d=$ $d_{\text {in }}=\lambda / 2$. We name $d$ the basic array spacing parameter of the 2LNA in this paper. In the same way, we assume that $M=M_{1}+M_{2}$ transmitting pulses with 2LN-PRI are received in a CPI for each range gate, where the pulse locations can be determined by the union of sets $Q_{\text {in }}$ and $Q_{\text {out }}$. For simplicity, we also represent the pulse positions using a new set $Q=$ $\left\{m T, m=0, m_{1}, m_{2}, \ldots, m_{M-1}\right\}$, where $T=T_{i n}$ is named the basic PRI parameter of the 2LN-PRI in this paper.

According to this radar configuration, the spatial steering vector can be given by

$$
\mathbf{a}\left(f_{s}\right)=\left[1, e^{j 2 \pi f_{s} n_{1}}, \ldots, e^{j 2 \pi f_{s} n_{N-1}}\right]^{T}
$$

where $[\bullet]^{T}$ denotes the transpose operation and $f_{s}$ is the spatial frequency which is given by

$$
f_{s}=\frac{d \sin (\theta)}{\lambda}
$$

where $\theta$ is the angle of arrival (AOA). At the same time, the Doppler steering vector $\mathbf{b}\left(f_{d}\right)$ can be written as

$$
\mathbf{b}\left(f_{d}\right)=\left[1, e^{j 2 \pi f_{d} m_{1}}, \ldots e^{j 2 \pi f_{d} m_{M-1}}\right]^{T}
$$

where $f_{d}$ is the normalized Doppler frequency given by

$$
f_{d}=\frac{2 v \sin (\theta) T}{\lambda}=\beta f_{s}
$$

where $\beta=2 v T / d$ and $v$ is the relative velocity between the moving radar platform and the signal source from direction 
$\theta . T=T_{\text {in }}$ denotes the basic PRI parameter of the 2LN-PRI. Then, the space-time steering vector $\mathbf{v}$ can be expressed as

$$
\mathbf{v}\left(f_{s}, f_{d}\right)=\mathbf{b}\left(f_{d}\right) \otimes \mathbf{a}\left(f_{s}\right)
$$

where $\otimes$ denotes the Kronecker product.

Suppose that $N_{c}$ clutter patches are uniformly distributed among the angles from 0 degrees to 360 degrees in a fixed range bin. We denote $\alpha_{i}, f_{s c}^{i}$, and $f_{d c}^{i}$ as the complex amplitude, the spatial frequency, and the normalized Doppler frequency of the $i$ th clutter patch, respectively. The received signal $\mathbf{x}_{c}$, which contains clutter $\mathbf{c}$ plus noise $\mathbf{n}$, can be expressed by

$$
\begin{aligned}
\mathbf{x}_{c} & =\mathbf{c}+\mathbf{n}=\sum_{i=1}^{N_{c}} \alpha_{i} \mathbf{b}\left(f_{d c}^{i}\right) \otimes \mathbf{a}\left(f_{s c}^{i}\right)+\mathbf{n}=\sum_{i=1}^{N_{c}} \alpha_{i} \mathbf{v}_{c i}+\mathbf{n} \\
& =\sum_{i=1}^{N_{c}} \mathbf{c}_{i}+\mathbf{n}
\end{aligned}
$$

where $\mathbf{v}_{c i}=\mathbf{v}\left(f_{s c}^{i}, f_{d c}^{i}\right)=\mathbf{b}\left(f_{d c}^{i}\right) \otimes \mathbf{a}\left(f_{s c}^{i}\right)$ and $\mathbf{c}_{i}$ denote the space-time steering vector and the returned signal of the $i$ th clutter patch, respectively. It is assumed that the clutter patches are mutually uncorrelated, and then the clutter covariance matrix (CCM) corresponding to the physical array and pulses can be given by [1-3]

$$
\mathbf{R}_{c}=\sum_{i=1}^{N_{c}} E\left[\mathbf{c}_{i} \mathbf{c}_{i}^{H}\right]=\sum_{i=1}^{N_{c}} \sigma_{i}^{2} \mathbf{v}_{c i} \mathbf{v}_{c i}^{H}=\mathbf{V}_{c} \mathbf{P} \mathbf{V}_{c}^{H}
$$

where $\mathbf{V}_{c}=\left[\mathbf{v}_{c 1}, \mathbf{v}_{c 2}, \ldots, \mathbf{v}_{c i}, \ldots, \mathbf{v}_{c N_{c}}\right]$ is the clutter spacetime steering matrix, $\sigma_{i}^{2}=E\left[\left|\alpha_{i}^{2}\right|\right]$ denotes the power of the $i$ th clutter patch, $\mathbf{P}=\operatorname{diag}\left(\left[\sigma_{1}^{2}, \sigma_{2}^{2}, \ldots, \sigma_{N_{c}}^{2}\right]^{T}\right)$ is a diagonal matrix, $[\bullet]^{H}$ is the conjugate transpose operation, and $E[\bullet]$ indicates the expectation operator. Additionally, the noises are also assumed to be mutually uncorrelated, and then the noise covariance matrix $\mathbf{R}_{n}$ is described as

$$
\mathbf{R}_{n}=\sigma_{n}^{2} \mathbf{I}_{M N}
$$

where $\sigma_{n}^{2}$ is the noise power and $\mathbf{I}_{M N}$ is an $M N \times M N$ identity matrix. Then, in the assumption that the clutter is independent of the noise, the CNCM can be given by

$$
\mathbf{R}_{u}=E\left[\mathbf{x}_{c} \mathbf{x}_{c}^{H}\right]=\mathbf{V}_{c} \mathbf{P} \mathbf{V}_{c}^{H}+\sigma_{n}^{2} \mathbf{I}_{M N}=\mathbf{R}_{c}+\mathbf{R}_{n} .
$$

We call this CNCM as the traditional CNCM in order to distinguish it from the virtual CNCM that will be presented in the later contents.

As is well known, denoting $f_{s t}$ and $f_{d t}$ as the spatial frequency and the normalized Doppler frequency of an interesting target, the traditional optimum adaptive filter weights of STAP can be given by [1]

$$
\mathbf{w}=\frac{\mathbf{R}_{u}^{-1} \mathbf{v}_{t}}{\sqrt{\mathbf{v}_{t}^{H} \mathbf{R}_{u}^{-1} \mathbf{v}_{t}}}
$$

where $\mathbf{v}_{t}=\mathbf{v}\left(f_{s t}, f_{d t}\right)=\mathbf{b}\left(f_{d t}\right) \otimes \mathbf{a}\left(f_{s t}\right)$ denotes the space-time steering vector of the interesting target and $\mathbf{R}_{u}^{-1}$ denotes the inverse matrix of $\mathbf{R}_{u}$. The term $\left(\mathbf{v}_{t}^{H} \mathbf{R}_{u}^{-1} \mathbf{v}_{t}\right)^{1 / 2}$ is set to satisfy the constant false alarm rate (CFAR) property. In addition, the traditional optimum output SINR can be given by [3]

$$
\operatorname{SINR}=\sigma_{t}^{2} \mathbf{v}_{t}^{H} \mathbf{R}_{u}^{-1} \mathbf{v}_{t}
$$

where $\sigma_{t}^{2}$ denotes the target power.

However, the true traditional CNCM is commonly unknown and needs to be estimated by the MLE approach using the training samples, which is given by [3]

$$
\widetilde{\mathbf{R}}_{u}=\frac{1}{K} \mathbf{X}_{c} \mathbf{X}_{c}^{H}
$$

where $\widetilde{\mathbf{R}}_{u}$ is the estimation of the traditional CNCM, $K$ denotes the number of training samples, and $\mathbf{X}_{c}$ indicates the training sample matrix formed with the training sample snapshots collected from $K$ range cells.

3.2. Derivation of Virtual Space-Time Snapshot. An approach to acquire virtual space-time snapshot was firstly reported in [30] for coprime arrays. Herein, we employ a similar process to derive the virtual space-time snapshot for the 2LNA with 2LN-PRI. Note that the term $\mathbf{v}\left(f_{s}, f_{d}\right) \mathbf{v}\left(f_{s}, f_{d}\right)^{H}$ can be rewritten as

$$
\begin{aligned}
\mathbf{v v}^{H} & =\left[\mathbf{b}\left(f_{d}\right) \otimes \mathbf{a}\left(f_{s}\right)\right]\left[\mathbf{b}\left(f_{d}\right) \otimes \mathbf{a}\left(f_{s}\right)\right]^{H} \\
& =\left[\mathbf{b}\left(f_{d}\right) \mathbf{b}\left(f_{d}\right)^{H}\right] \otimes\left[\mathbf{a}\left(f_{s}\right) \mathbf{a}\left(f_{s}\right)^{H}\right],
\end{aligned}
$$

owing to the property of $(A \otimes B)(C \otimes D)=(A C) \otimes(B D)$. Moreover, the $(p, q)$ th entry in $\mathbf{a}\left(f_{s}\right) \mathbf{a}\left(f_{s}\right)^{H}$ has the form

$$
\left[\mathbf{a}\left(f_{s}\right) \mathbf{a}\left(f_{s}\right)^{H}\right]_{p, q}=e^{j 2 \pi f_{s}\left(n_{p}-n_{q}\right)}=e^{j 2 \pi f_{s} \widehat{n}_{p q}} .
$$

We can observe that (20) actually reflects the difference coarray of a 2LNA according to the definition in (2). Then, for the considered 2LNA whose difference coarray is an ULA with $\widehat{N}$ elements, the set $\left\{\widehat{n}_{p q}, p, q=0, n_{1}, \cdots n_{N-1}\right\}$ in (20) should contain all integers from $-N_{c o}$ to $N_{c o}$ according to (3). And we define a new set $\{\hat{n}\}$ which consists of the distinct elements of the set $\left\{\widehat{n}_{p q}\right\}$. Then, there exists an arrangement that could convert $\mathbf{a}\left(f_{s}\right) \mathbf{a}\left(f_{s}\right)^{H}$ to a new spatial steering vector $\widehat{\mathbf{a}}\left(f_{s}\right)$, which is corresponding to a virtual ULA with $\widehat{N}$ elements and $\widehat{n} d$ spacing in the coarray domain. $\widehat{\mathbf{a}}\left(f_{s}\right)$ can be written as

$$
\widehat{\mathbf{a}}\left(f_{s}\right)=\left[e^{-j 2 \pi f_{s} N_{c o}}, \cdots 1, \ldots, e^{j 2 \pi f_{s} N_{c o}}\right]^{T} .
$$

Similarly, the $(k, l)$ th entry in $\mathbf{b}\left(f_{d}\right) \mathbf{b}\left(f_{d}\right)^{H}$ takes the form

$$
\left[\mathbf{b}\left(f_{d}\right) \mathbf{b}\left(f_{d}\right)^{H}\right]_{k, l}=e^{j 2 \pi f_{d}\left(m_{k}-m_{l}\right)}=e^{j 2 \pi f_{d} \widehat{m}_{k l}}
$$

where the set $\left\{\widehat{m}_{k l}, k, l=0, m_{1}, \cdots m_{M-1}\right\}$ contains all integers from $-M_{c o}$ to $M_{c o}$ in the condition of 2LN-PRI. Still defining a new set $\{\widehat{m}\}$ which consists of the distinct elements 
of the set $\left\{\widehat{m}_{k l}\right\}$, the term $\mathbf{b}\left(f_{d}\right) \mathbf{b}\left(f_{d}\right)^{H}$ can be rearranged into a new Doppler steering vector $\widehat{\mathbf{b}}\left(f_{d}\right)$, which is corresponding to $\widehat{M}$ virtual pulses with constant PRI $\widehat{m} T$ in a CPI and can be expressed as

$$
\widehat{\mathbf{b}}\left(f_{d}\right)=\left[e^{-j 2 \pi f_{d} M_{c o}}, \cdots 1, \ldots, e^{j 2 \pi f_{d} M_{c o}}\right]^{T} .
$$

Furthermore, it is noted that the expression (15) of CNCM $\mathbf{R}_{u}$ includes the term $\mathbf{v}_{c i} \mathbf{v}_{c i}^{H}$. Thus, based on the analysis shown from (19) to (23), it is possible to derive a virtual space-time data snapshot corresponding to a virtual ULA and virtual pulses with constant PRI from the expression (15). We can extract the specified elements in the matrix $\mathbf{R}_{u}$ to construct a new $\widehat{N} \times \widehat{M}$ matrix $\mathbf{Z}$ such that

$$
\mathbf{Z}=\sum_{i=1}^{N_{c}} \sigma_{i}^{2} \widehat{\mathbf{a}}\left(f_{s c}^{i}\right) \widehat{\mathbf{b}}\left(f_{d c}^{i}\right)^{T}+\sigma_{n}^{2} \mathbf{e}_{1} \mathbf{e}_{2}^{T}
$$

where $\mathbf{e}_{1}$ and $\mathbf{e}_{2}$ are $\widehat{N} \times 1$ and $\widehat{M} \times 1$ column vectors of all zeros except a one at center position, respectively. Thus, a $\widehat{M} \widehat{N} \times 1$ virtual space-time snapshot of the clutter plus noise can be got through vectoring (24), which can be expressed as

$$
\begin{aligned}
\mathbf{z} & =\sum_{i=1}^{N_{c}} \sigma_{i}^{2} \widehat{\mathbf{b}}\left(f_{d c}^{i}\right) \otimes \widehat{\mathbf{a}}\left(f_{s c}^{i}\right)+\sigma_{n}^{2} \mathbf{e}_{2} \otimes \mathbf{e}_{1}=\sum_{i=1}^{N_{c}} \sigma_{i}^{2} \widehat{\mathbf{v}}_{c i}+\widehat{\mathbf{n}} \\
& =\widehat{\mathbf{c}}+\widehat{\mathbf{n}}
\end{aligned}
$$

where $\widehat{\mathbf{v}}_{c i}=\widehat{\mathbf{b}}\left(f_{d c}^{i}\right) \otimes \widehat{\mathbf{a}}\left(f_{s c}^{i}\right)$ denotes the virtual space-time steering vector of the $i$ th clutter patch. $\widehat{\mathbf{c}}$ and $\widehat{\mathbf{n}}$ indicate the virtual clutter vector and the virtual noise vector, respectively.

Comparing (25) with (12), we can find that $\mathbf{z}$ behaves like a virtual clutter plus noise signal collected from a virtual ULA with $\widehat{N}$ elements and $\widehat{n} d$ spacing in a virtual CPI with $\widehat{M}$ uniform transmitting pulses with time intervals $\widehat{m} T$. But the differences are that the amplitudes of the virtual clutter patches are indeed the powers of the realistic clutter patches and the virtual noise becomes a deterministic vector which is also determined by the power of the realistic noise. Moreover, it is noted that, under the constraints of $N=N_{1}+N_{2}$ and $M=M_{1}+M_{2}$, the inequalities that $\widehat{N}>N$ and $\widehat{M}>M$ are always maintained whatever the values of $\left(N_{1}, N_{2}\right)$ and $\left(M_{1}, M_{2}\right)$ are. That is to say, the virtual spacetime snapshot $\mathbf{z}$ has an increased number of DOFs in contrast with the physical one as shown in (12). Thus, it is attractive to implement STAP on the virtual space-time snapshot $\mathbf{z}$ for improving the performance of clutter suppression and target detection. However, in view of the fact that $\mathbf{z}$ is just a single snapshot, the corresponding $\mathrm{CNCM}$ estimation in term of expression (18) is a rank-one matrix, which is hard to be used to design the STAP filter $[2,3]$. In order to build a full-rank CNCM estimation, we make the spatial-temporal smoothing technique operate on the virtual space-time snapshot to get a virtual CNCM estimation for STAP filter. The detailed process will be presented in Section 3.3.

3.3. Derivation of Virtual CNCM Estimation. For applying the spatial-temporal smoothing technique, the data matrix

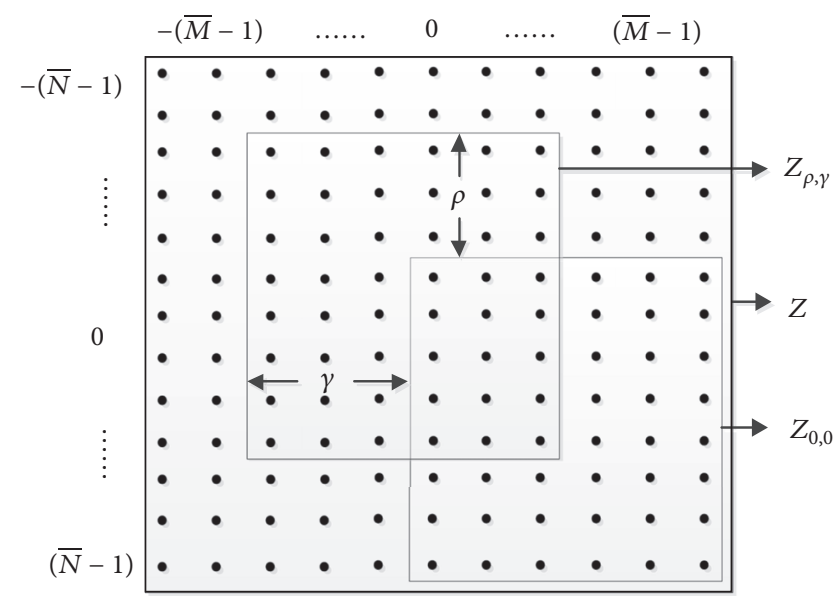

FIGURE 3: Partition strategy of the virtual space-time snapshot.

$\mathrm{Z}$ should be divided into $\bar{M} \bar{N}$ number of submatrixes, each with size $\bar{N} \times \bar{M}$, where $\bar{N}=N_{2}\left(N_{1}+1\right)$ and $\bar{M}=M_{2}\left(M_{1}+\right.$ $1)$. The partition strategy is illustrated in Figure 3 , and the submatrix can be defined as

$$
\mathbf{Z}_{\rho, \gamma}=\sum_{i=1}^{N_{c}} e^{-j\left(\rho f_{s c}^{i}+\gamma f_{d c}^{i}\right)} \sigma_{i}^{2} \overline{\mathbf{a}}\left(f_{s c}^{i}\right) \overline{\mathbf{b}}\left(f_{d c}^{i}\right)^{T}+\sigma_{n}^{2} \mathbf{e}_{1, \rho} \mathbf{e}_{2, \gamma}^{T}
$$

where $\rho=0,1, \ldots, \bar{N}-1, \gamma=0,1, \ldots, \bar{M}-1, \mathbf{e}_{1, \rho}$ is a subvector constructed from the $(\bar{N}-\rho)$ th entry to the $(2 \bar{N}-1-\rho)$ th entry of $\mathbf{e}_{1}, \mathbf{e}_{2, \gamma}$ is a subvector constructed from the $(\bar{M}-\gamma)$ th entry to the $(\bar{M}-\gamma)$ th entry of $\mathbf{e}_{2}, \overline{\mathbf{a}}\left(f_{s c}^{i}\right)$ is the spatial steering vector corresponding to a virtual sub-ULA with $\bar{N}$ elements and basic spacing $d$, and $\overline{\mathbf{b}}\left(f_{d c}^{i}\right)$ is the Doppler steering vector corresponding to $\bar{M}$ virtual pulses in a sub-CPI with basic PRI parameter $T$.

Then, we can define a new covariance matrix via spatialtemporal smoothing approach as follows:

$$
\mathbf{R}_{s}=\frac{1}{\bar{M}} \bar{N} \sum_{\rho=0}^{\bar{N}-1} \sum_{\gamma=0}^{\bar{M}-1} \mathbf{z}_{\rho, \gamma} \mathbf{z}_{\rho, \gamma}^{H}
$$

where $\mathbf{z}_{\rho, \gamma}=\operatorname{vec}\left(\mathbf{Z}_{\rho, \gamma}\right)$ and $\operatorname{vec}(\bullet)$ means to transform a matrix into a column vector. It is noted that $\mathbf{R}_{s}$ is a Hermitian positive semidefinite matrix of size $\bar{M} \bar{N} \times \bar{M} \bar{N}$. Following the same proof process of Theorem 2 in [22] or Theorem 3 in [34], the expression (27) can be reformulated as

$$
\mathbf{R}_{s}=\frac{1}{\bar{M} \bar{N}} \overline{\mathbf{R}}_{u}^{2}
$$

where

$$
\overline{\mathbf{R}}_{u}=\overline{\mathbf{V}}_{c} \mathbf{P} \overline{\mathbf{V}}_{c}^{H}+\sigma_{n}^{2} \mathbf{I} \bar{M} \bar{N}
$$

where $\overline{\mathbf{V}}_{c}=\left[\overline{\mathbf{v}}_{c 1}, \overline{\mathbf{v}}_{c 2}, \ldots, \overline{\mathbf{v}}_{c N_{c}}\right], \overline{\mathbf{v}}_{c i}=\overline{\mathbf{b}}\left(f_{d c}^{i}\right) \otimes \overline{\mathbf{a}}\left(f_{s c}^{i}\right)$, and $\mathbf{P}$ is the diagonal matrix which represents the powers of all clutter patches as aforementioned in Section 3.1. Then, like the expression (15), $\overline{\mathbf{R}}_{u}$ can be exactly viewed as the virtual 
CNCM estimation of the signals received from a virtual subULA with $\bar{N}$ antenna elements and $\bar{M}$ virtual transmitting pulses in a sub-CPI with constant PRI. $\overline{\mathbf{v}}_{c i}$ is regarded as another virtual space-time steering vector corresponding to the sub-ULA and sub-CPI. On the other hand, it is hard to calculate $\overline{\boldsymbol{R}}_{u}$ via (29) in practice because we can hardly get the precise $f_{d c}^{i}, f_{s c}^{i}$, and $\sigma_{i}^{2}$ of the $i$ th clutter patch direct from the echoes to compute the matrixes $\overline{\mathbf{V}}_{c}$ and $\mathbf{P}$. Contrarily, since $\mathbf{R}_{s}$ can be easily computed by a sum of vector outer products with finite snapshots as presented in (27), $\overline{\mathbf{R}}_{u}$ is suggested to be calculated by (28), which can be rewritten as

$$
\overline{\mathbf{R}}_{u}=\left(\bar{M} \bar{N} \mathbf{R}_{s}\right)^{1 / 2}
$$

Thus, as is described in Section 3.4, the matrix $\overline{\mathbf{R}}_{u}$ can be employed as the CNCM estimation for O2LN-STAP filter.

3.4. STAP Filter. Finally, we can perform the STAP filter based on the virtual sub-ULA with $\bar{N}$ antenna elements and the $\bar{M}$ virtual transmitting pulses in a sub-CPI with constant PRI. Then, the corresponding virtual optimum adaptive filter weights can be given by

$$
\overline{\mathbf{w}}=\frac{\overline{\mathbf{R}}_{u}^{-1} \overline{\mathbf{v}}_{t}}{\sqrt{\overline{\mathbf{v}}_{t}^{H} \overline{\mathbf{R}}_{u}^{-1} \overline{\mathbf{v}}_{t}}}
$$

where $\overline{\mathbf{v}}_{t}=\overline{\mathbf{b}}\left(f_{d t}\right) \otimes \overline{\mathbf{a}}\left(f_{s t}\right)$ denotes the virtual target spacetime steering vector with respect to the sub-ULA and subCPI and $\overline{\mathbf{R}}_{u}^{-1}$ denotes the inverse matrix of $\overline{\mathbf{R}}_{u}$. Note that the expression (31) is actually acquired by substituting $\overline{\mathbf{R}}_{u}$ and $\overline{\mathbf{v}}_{t}$ for $\mathbf{R}_{u}$ and $\mathbf{v}_{t}$ in (16), respectively. Additionally, the virtual optimum output SINR corresponding to the sub-ULA and sub-CPI can be written as

$$
\operatorname{VSINR}=\sigma_{t}^{2} \overline{\mathbf{v}}_{t}^{H} \overline{\mathbf{R}}_{u}^{-1} \overline{\mathbf{v}}_{t}
$$

Significantly, using the space-time two-level nested structure, the final available DOFs for STAP filter are indeed equivalent to $\bar{M} \bar{N}$ rather than $\widehat{M} \widehat{N}$. Intuitively, we rewrite the value of DOFs again as follows:

$$
D_{2-\text { nested }}=\bar{M} \bar{N}=M_{2}\left(M_{1}+1\right) \times N_{2}\left(N_{1}+1\right)
$$

Remarkably, though $\bar{N}<\widehat{N}$ and $\bar{M}<\widehat{M}$, the inequalities that $\bar{N} \geq N$ and $\bar{M} \geq M$ are still maintained under the constraints of $N=N_{1}+N_{2}$ and $M=M_{1}+M_{2}$. In other words, the available DOFs of the STAP with the space-time two-level nested structure are still larger than that of the traditional approaches. Using $\overline{\mathbf{w}}$ for STAP filter is able to get more DOFs than using $\mathbf{w}$ that is directly derived from the physical antenna array and transmitting pulses:

$$
\begin{aligned}
& D_{2-\text { nested }}^{\text {opt }} \\
& = \begin{cases}\frac{(N+1)^{2}}{4} \times{\frac{(M+1)^{2}}{4}}^{2} & N \text { : odd } ; M: \text { odd } ;(a) \\
\left(\frac{N^{2}}{4}+\frac{N}{2}\right) \times\left(\frac{M^{2}}{4}+\frac{M}{2}\right) & N \text { : even; } M \text { : even } ;(b) \\
\frac{(N+1)^{2}}{4} \times\left(\frac{M^{2}}{4}+\frac{M}{2}\right) & N: \text { odd } ; M: \text { even } ;(c) \\
\left(\frac{N^{2}}{4}+\frac{N}{2}\right) \times \frac{(M+1)^{2}}{4} & N: \text { even; } M: \text { odd } ;(d)\end{cases}
\end{aligned}
$$

In particular, in the case of O2LNA and O2LN-PRI, the maximum available DOFs can be given by expression (34), which is able to generate $O\left(M^{2} N^{2}\right)$ DOFs from $O(M N)$ physical elements. Then, the STAP strategy using O2LNA and $\mathrm{O} 2 \mathrm{LN}-\mathrm{PRI}$ is referred to as O2LN-STAP.

\section{Results and Discussion}

In this section, numerical experiments and results are offered to verify the theoretical derivations and compare the performances of the O2LN-STAP with those of the traditional uniform-STAP and the recently reported coprime-STAP. We assume a side-looking airborne radar composed of an O2LNA with $N_{1}=N_{2}=3$ and an O2LN-PRI with $M_{1}=$ $M_{2}=3$. Obviously, the total numbers of array elements and transmitting pulses in a CPI are $N=6$ and $M=6$, respectively. The carrier frequency is $10 \mathrm{GHz}$, the basic PRI is $T=1 / 600 \mathrm{~Hz}$, and the basic array element spacing is $d=\lambda / 2$. Without loss of generality, we set $\beta=1$ to avoid temporal aliasing. There are 180 clutter patches uniformly distributed in the forward area of the antenna array, which are assumed to follow the zero-mean complex-valued Gaussian distributions. The noise power is normalized to $0 \mathrm{~dB}$ and the clutter-to-noise ratio (CNR) is set to $30 \mathrm{~dB}$. It is assumed that a target is located at angle 16.5 degrees, and its normalized Doppler frequency is -0.27 .

4.1. Smoothed Virtual CNCM Estimation. In STAP applications, the eigenspectrum is usually utilized to measure the similarity of different covariance matrices. Herein, we also employ it to validate the derivation of virtual CNCM estimation from the spatial-temporal smoothing technique. According to the simulation parameters, we can get that $\bar{N}=$ 12 and $\bar{M}=12$. We define a new matrix $\mathbf{R}_{v}$ to represent the theoretical CNCM of an ULA with $\bar{N}$ elements and $\bar{M}$ pulses, where the clutter space-time steering vectors and amplitudes are both assumed to be priorly known. In other words, the matrix $\mathbf{R}_{v}$ is able to be calculated via (29). Thus, we compare $\overline{\mathbf{R}}_{u}$, which is computed through (30), with $\mathbf{R}_{v}$ and $\mathbf{R}_{s}$ in terms of their eigenspectra. Figure 4 depicts the comparative results. According to the Brennan rule [3], the rank of $\mathbf{R}_{v}$ should be $\bar{N}+\beta(\bar{M}-1)=23$. And we can see from the figure that $\overline{\mathbf{R}}_{u}$ and $\mathbf{R}_{s}$ have the same rank as $\mathbf{R}_{v}$. But the eigenvalues of $\mathbf{R}_{s}$ are very different from the others, while the eigenvalues of $\overline{\mathbf{R}}_{u}$ and $\mathbf{R}_{v}$ are nearly overlapped completely. Thus, we can see that $\overline{\mathbf{R}}_{u}$ has the same eigenspectrum as $\mathbf{R}_{v}$. Therefore, on the basis of these eigenspectra, we have demonstrated the theoretical derivation of $\overline{\mathbf{R}}_{u}$, which is indeed matching to a virtual ULA 


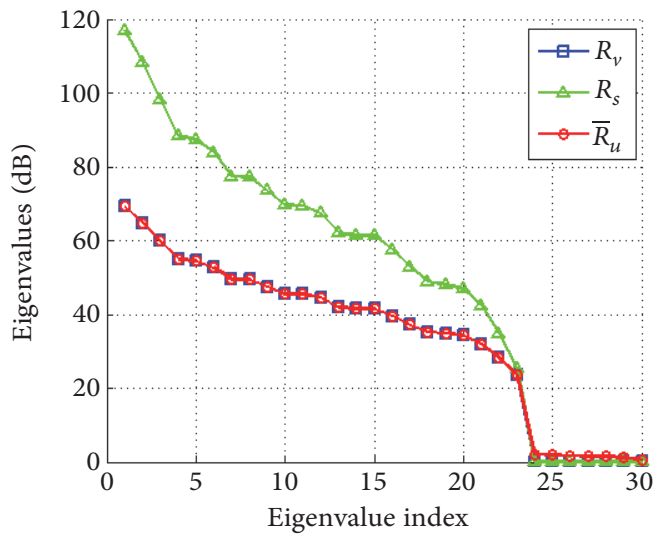

FIGURE 4: Eigenspectra of different covariance matrices.

with $\bar{N}$ antenna elements and $\bar{M}$ virtual transmitting pulses in a CPI with constant PRI, as is expressed in (29) and (30).

4.2. Performance Comparison with Traditional Uniform-STAP. As we know, the traditional uniform-STAP is commonly based on the ULA with uniform PRI. In addition, we will take another method named as direct-nested-STAP into consideration too, which adopts the same direct processing as the traditional uniform-STAP but acts on the physical O2LNA and pulses with O2LN-PRI. In this subsection, we compare the performance of the O2LN-STAP with the traditional uniform-STAP and the direct-nested-STAP in two different conditions: one is setting a fixed number of physical array elements and transmitting pulses; another is setting a fixed objective of DOFs.

(1) Setting a Fixed Number of Physical Array Elements and Pulses. Firstly, we implement the comparison under the restriction of fixed numbers of physical array elements $N$ and transmitting pulses $M$. Without loss of generality, we suppose that $N$ and $M$ are even numbers. For O2LN-STAP, we can get that $N_{1}=N_{2}=N / 2$ and $M_{1}=M_{2}=M / 2$, as is described in Proposition 1 . And the final available DOFs value for the O2LN-STAP is given by (34)(b). In contrast, it is well known that the available DOFs of the traditional uniformSTAP and the direct-nested-STAP are only equivalent to $M N$, which is evidently much smaller than the value of $(34)(\mathrm{b})$. For example, fixing $N=6$ and $M=6$, the value of (34)(b) is 144 , but $M N$ equals to 36 .

Furthermore, we study the normalized STAP filter output response and the optimum output SINR performance to illustrate the advantages of the O2LN-STAP in the case of fixed number of array elements and transmitting pulses. Figure 5 presents the normalized STAP filter output response for the three different STAP strategies. Specifically, Figures 5(a), 5(b), and 5(c) are corresponding to the O2LN-STAP, the traditional uniform-STAP, and the direct-nested-STAP, respectively. It is observed that all of the three strategies can form a deep notch at the clutter ridge and make a maximum peak at the target position. Nevertheless, the side-lobe levels and the angle-Doppler resolution of the O2LN-STAP are better than those of the other two approaches owing to the increased available DOFs of the O2LN-STAP. Moreover, the direct-nested-STAP even shows much higher side-lobe levels than the traditional uniform-STAP obviously. It is because that though the direct-nested-STAP seems to utilize the nested array and pulses too, it actually fails to construct a larger virtual ULA and more virtual pulses with uniform PRI. The direct-nested-STAP also just directly operates on the physical nested array and pulses rather than takes advantage of the difference coarray and copulses. Therefore, the sparse property of the nested array and pulses results in the poor side-lobe performance of the direct-nested-STAP.

In addition, in the condition of fixed number of array elements and transmitting pulses, the optimum output SINR curves against the target normalized Doppler frequency are shown in Figure 6. The SINR curve of the O2LN-STAP is derived from (32), and the others are from (17). It is clear that the SINR of the O2LN-STAP in the nonclutter regions is much higher than those of the traditional uniformSTAP and the direct-nested-STAP owing to the enhanced DOFs of O2LN-STAP. Moreover, the O2LN-STAP method and the direct-nested-STAP both possess a narrower clutter notch than the traditional uniform-STAP, which means an improvement of MDV performance. However, in view of the bad filter output response as shown in Figure 5(c), we do not suggest to use the direct-nested-STAP for improving the MDV performance in practice.

Note that almost all conventional STAP methods employ constant uniform PRI. But the electronic countercountermeasures (ECCM) capabilities are restricted by the uniform PRI. Moreover, in contrast with the uniform PRI, the two-level nested PRI approach can get a comparative performance with the uniform PRI approach that has a larger number of physical pulses. In other words, the twolevel nested PRI approach is potential to reduce the radar probability of intercept owing to the low accumulation of transmitting energy and time. Therefore, the two-level nested PRI approach can get some advantages over the conventional way in these aspects. On the other hand, the small number of pulses ought to cause a performance decrease (e.g., the decrease of Doppler resolution and high side-lobe levels in temporal dimension) as shown in Figure 5(b). However, we can obtain an increased number of virtual pulses via the transformation described in Section 3 to offset the deficiency of the physical pulses for performance improvements, as is presented in Figure 5(a). Additionally, from the hardware perspective, the conventional uniform PRI may be easier to realize. Nevertheless, the two-level nested PRI approach is also not hard to realize for the reason that the two-level nested structure is actually thinned from the conventional uniform one and close to the uniform structure; besides, it may get several benefits in contrast with the conventional way.

With respect to the other situations that $N$ and $M$ are not even integers, similar analysis procedures and simulation results can also be carried out to confirm the effectiveness of the O2LN-STAP, which will not be presented here on account of the space limitation. In summary, we can draw a conclusion that (which is referred to as conclusion 1 in the following), given a fixed number of physical array elements 


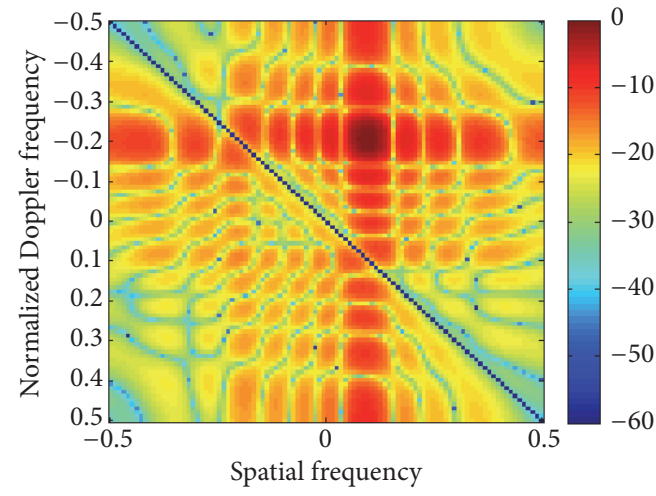

(a)

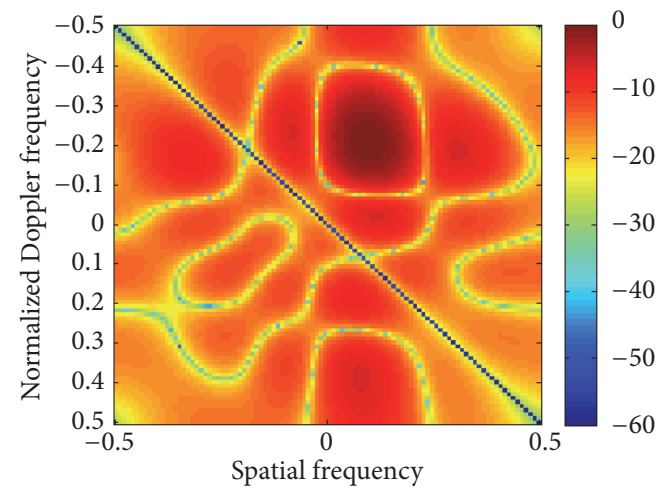

(b)

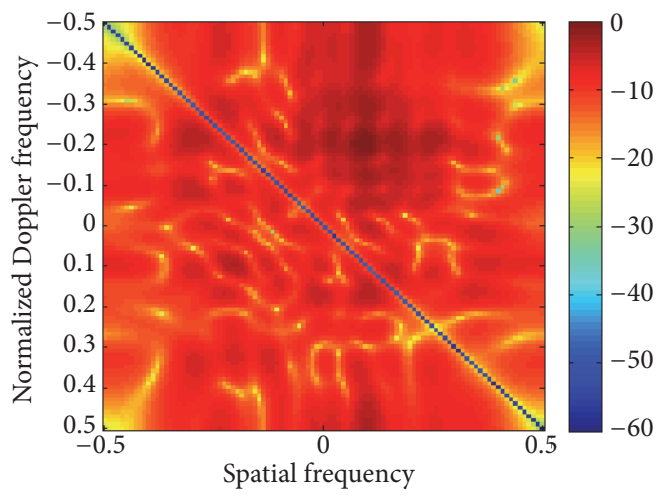

(c)

FIGURE 5: Normalized STAP filter output responses in the case of fixed total number of array elements and transmitting pulses. (a) The proposed O2LN-STAP. (b) The traditional uniform-STAP. (c) The direct-nested-STAP.

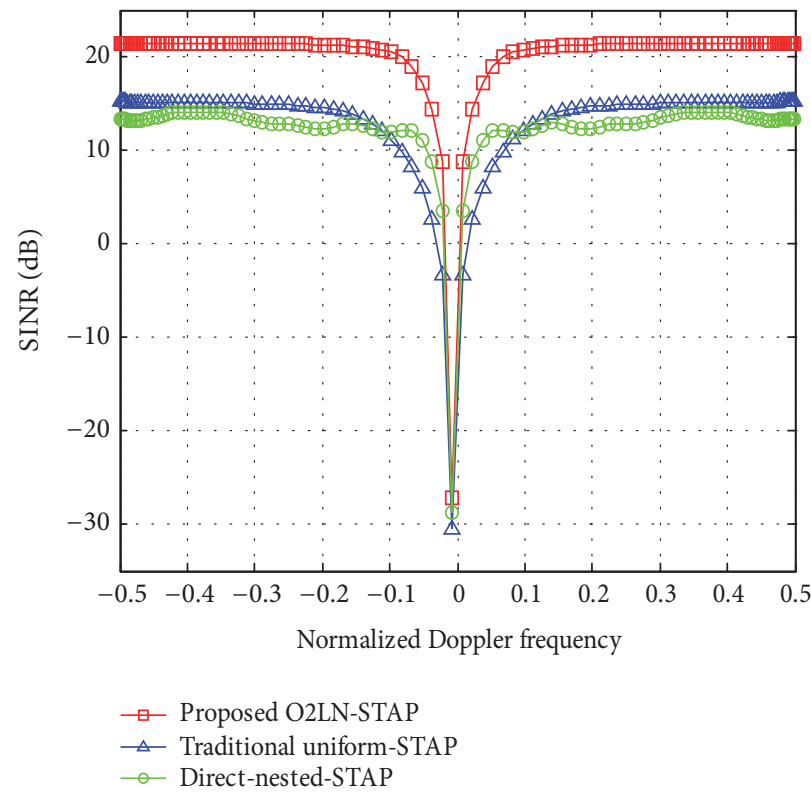

FIGURE 6: Optimum output SINR performances against target normalized Doppler frequency with $\mathrm{N}=6$ array elements and $\mathrm{M}=6$ transmitting pulses. 
and transmitting pulses, the O2LN-STAP can obtain more DOFs than the traditional uniform-STAP and the directnested-STAP, which improves the filter output response, SINR and MDV performances.

(2) Setting a Fixed Objective of DOFs. In most of the STAP applications, we prefer to achieve a fixed objective of DOFs using as low cost as possible for performance improvement. Remarkably, the converse of conclusion 1 means that the O2LN-STAP needs fewer physical array elements and transmitting pulses than the other two methods to achieve a certain designated number of DOFs. Considering that the direct-nested-STAP is not suitable for the practical applications due to its high side-lobe levels, we mainly focus on the comparison between O2LN-STAP and the traditional uniform-STAP in this subsection. For instance, if the objective of DOFs is set to 144, the O2LN-STAP can reach it with $N=M=6$, but the traditional uniform-STAP needs the configuration of $N=M=12$. Thereby, the O2LN-STAP method is potential to save the hardware resource and reduce the power consumption.

Furthermore, as is deduced in Section 3, we note that the O2LN-STAP method originates from the traditional CNCM $\mathbf{R}_{u}$. However, the true traditional CNCM is commonly unknown and needs to be estimated by the MLE approach using the training samples, as is described in (18). The accuracy of the estimation of the traditional CNCM is proportional to the ratio of the number of training samples $K$ to the product between the number of array elements and the number of transmitting pulses (i.e., $M N$ ) according to the $\mathrm{RMB}$ rule [3]. It is also well known that, with the increase of the number of training samples, the estimation error of the traditional CNCM for small $M N$ would converge to zero more quickly than that for large $M N$. Therefore, using CNCM estimation to replace the true CNCM for STAP filter would result in SINR loss due to the estimation error. Note that the O2LN-STAP could achieve an objective of DOFs with smaller $M N$ than the traditional uniform-STAP. Hence, it seems that the output SINR of the O2LN-STAP may converge to an identical optimum level more rapidly than that of the traditional uniform-STAP since the O2LN-STAP employs fewer physical array elements and transmitting pulses. That is to say, it is supposed that the O2LN-STAP may achieve a specific output SINR with smaller number of training samples than the traditional uniform-STAP, which is referred to as supposition 1 in the following.

In order to further study this issue, we carry out an experiment to analyze the convergence performance of the output SINR versus the number of training samples by the way of substituting the CNCM estimation for the true CNCM $\mathbf{R}_{u}$ to realize the deducing processing shown in Section 3 and then get the corresponding estimations of $\mathbf{R}_{s}$ and $\overline{\mathbf{R}}_{u}$ via (27) and (30) for the final filter processing, respectively. Note that we use the CNCM estimation in (18) that is actually acquired by the sample matrix inverse (SMI) algorithm [1] and the CNCM estimation calculated through principal components (PC) technique [3] to replace the true CNCM $\mathbf{R}_{u}$, respectively. This experiment is applied to both the O2LN-STAP and the traditional uniform-STAP for comparison. The results are

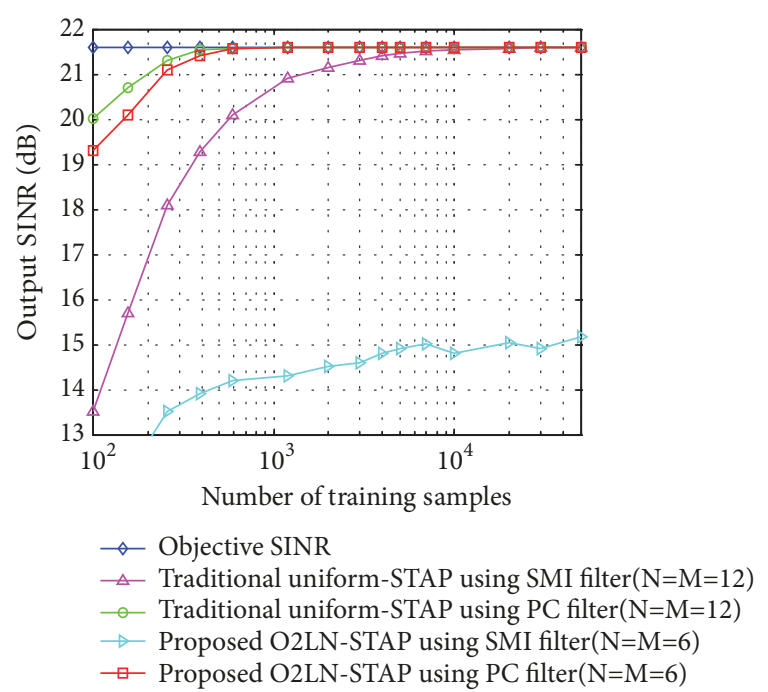

FIGURE 7: Output SINR performances versus the number of training samples.

provided in Figure 7. In the following, the term "using SMI filter" is corresponding to the aforementioned SMI approach and "using PC filter" is matching to the PC approach. The objective SINR curve in the figure is actually the theoretical upper bound of the output SINR corresponding to the traditional uniform-STAP with $N=M=12$. It can be seen that the O2LN-STAP using SMI filter has not converged to the objective SINR, while the others have reached it. Additionally, the approaches using PC filter present better convergence performance than those using SMI filter as expected, since the PC algorithm utilizes the clutter subspace technique to eliminate a part of cross correlation among clutter patches and then lower the requirement for training samples [3]. Moreover, the traditional uniform-STAP using PC filter (or SMI filter) exhibits faster convergence property and higher SINR in the case of a small number of training samples than the O2LN-STAP using PC filter (or SMI filter), which is not coincident with supposition 1 proposed in the preceding paragraph. This is because that, for the O2LNSTAP method, the estimation error of $\mathbf{R}_{u}$ would propagate throughout the additional procedures for the derivations of virtual space-time snapshot and virtual CNCM estimation, and the spatial-temporal smoothing process would also bring about a new estimation error. Therefore, we need more training samples to reduce this effect of error accumulation that is not existent in the traditional uniform-STAP. But from another point of view, we can see from Figure 7 that only employing half of the array elements and transmitting pulses of the traditional uniform-STAP, the O2LN-STAP using PC filter can achieve the specified aim of SINR with a close convergence performance to the traditional uniform-STAP using PC filter. On the other hand, the O2LN-STAP using $\mathrm{PC}$ filter still has a superior convergence performance than the traditional uniform-STAP using SMI filter. It is because that the requirement of sample numbers for PC technique is less than that for SMI approach [3], which makes the estimation error of $\mathbf{R}_{u}$ for PC technique become smaller than 


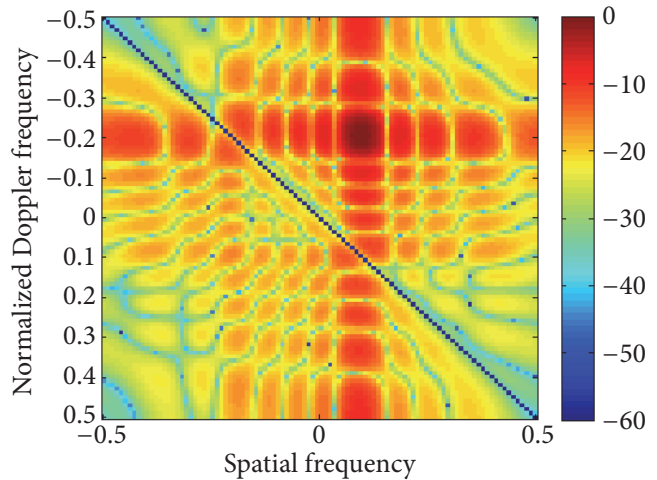

(a)

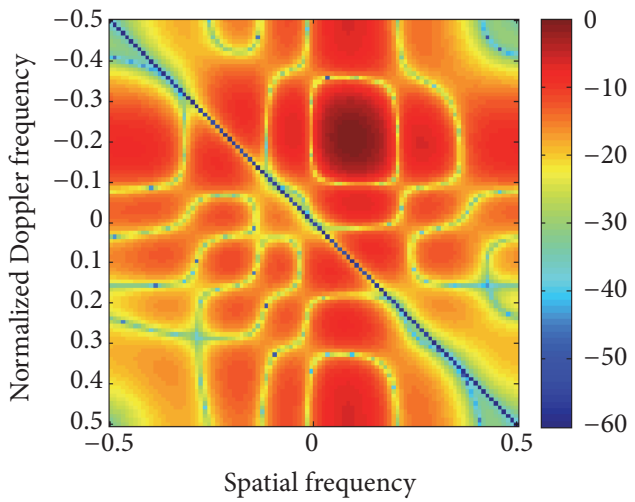

(b)

FIGURE 8: Normalized STAP filter output responses with $\mathrm{N}=6$ array elements and $\mathrm{M}=6$ transmitting pulses. (a) The proposed O2LN-STAP. (b) The coprime-STAP.

that for SMI approach. Therefore, using fewer array elements and pulses, the O2LN-STAP is still potential to be used for clutter suppression with less hardware complexity and power consumption.

4.3. Performance Comparison with Coprime-STAP. As aforementioned in the introduction section, the recently reported coprime-STAP strategy can also achieve enhanced DOFs to improve the STAP performance with low cost in contrast with the traditional uniform-STAP [32]. In this subsection, we will further compare the performance of the O2LN-STAP with that of the coprime-STAP. Herein, the coprime-STAP model used for analysis and simulation is identical with those in $[30,32]$. It is assumed that the spatial coprime array is composed of two ULA with interelement spacing $N_{1}^{\prime} d$ and $N_{2}^{\prime} d$, respectively, where $N_{1}^{\prime}$ and $N_{2}^{\prime}$ are coprime integers and satisfy $N_{1}^{\prime}<N_{2}^{\prime}$. Meanwhile, we also assume that the coprime PRI is a combination of two uniform PRIs denoted as $M_{1}^{\prime} T$ and $M_{2}^{\prime} T$, where $M_{1}^{\prime}$ and $M_{2}^{\prime}$ are coprime integers and satisfy $M_{1}^{\prime}<M_{2}^{\prime}$. Then, there are $N^{\prime}=N_{2}^{\prime}+2 N_{1}^{\prime}-1$ array elements and $\mathrm{M}^{\prime}=M_{2}^{\prime}+2 M_{1}^{\prime}-1$ pulses in a CPI in total for coprime-STAP. According to [30], the final available DOFs of the coprime-STAP can be given by

$$
D_{\text {coprime }}=\left(N_{1}^{\prime} N_{2}^{\prime}+1\right) \times\left(M_{1}^{\prime} M_{2}^{\prime}+1\right) .
$$

We also compare the performance of the O2LN-STAP with the coprime-STAP in the two different conditions as described in Section 4.2.

(1) Setting a Fixed Number of Physical Array Elements and Pulses. We first make the comparison in the case of a fixed number of physical array elements and transmitting pulses. We give a new proposition with respect to $D_{\text {coprime }}$ and $D_{2-\text { nested }}^{\text {opt }}$ under this situation, which is described as Proposition 3 in the following.

Proposition 3. Under the constraint of the same number of physical antenna array elements and transmitting pulses (i.e., $N=N^{\prime}, M=M^{\prime}$ ), the final available DOFs of the
O2LN-STAP $D_{2-n e s t e d}^{\text {opt }}$ is larger than that of the coprime-STAP $D_{\text {coprime }}$ (i.e., $D_{2-\text { nested }}^{\text {opt }}>D_{\text {coprime }}$ ), when $N>2$ and $M>2$.

Significantly, the conditions in Proposition 3 that $N>$ 2 and $M>2$ are almost always met in the practical applications. The detailed proof process can be found in appendix. For example, fixing $N=6$ array elements and $M=6$ transmitting pulses, the parameters of coprime-STAP have to be set as $N_{1}^{\prime}=M_{1}^{\prime}=2$ and $N_{2}^{\prime}=M_{2}^{\prime}=3$ for achieving a maximum value of DOFs, while the parameters of O2LNSTAP are set as $N_{1}=M_{1}=3$ and $N_{2}=M_{2}=3$. Then, we can get $D_{\text {coprime }}=49$ via (35), while $D_{2 \text {-nested }}^{\text {opt }}=144$ through (34)(b). Their normalized STAP filter output responses and optimum output SINR curves are illustrated in Figures 8 and 9 , respectively. It is clearly observed that, with the same number of physical array elements and transmitting pulses, the O2LN-STAP gains better angle-Doppler resolution, lower side-lobe levels, higher SINR, and narrower clutter notch (improved MDV performance) than the coprime-STAP.

(2) Setting a Fixed Objective of DOFs. We then compare the performances in the situation of a fixed objective of DOFs. Similar to the analysis in the previous subsection, it is noted that Proposition 3 can be illustrated in another way. That is to say, given a specified objective of DOFs, the O2LNSTAP can reach it with fewer physical array elements and transmitting pulses than the coprime-STAP. For example, we first assume that there exists a virtual space-time system consisting of a virtual ULA with 16 elements and 16 virtual transmitting pulses with uniform PRI in a CPI (this virtual space-time system is denoted as V-ULA-UPRI-16x16), whose DOFs equal to $16 \times 16=256$. Then, in order to construct an identical virtual space-time structure and obtain the same DOFs as V-ULA-UPRI-16x16, we can employ the O2LNSTAP with the configurations of $N_{1}=M_{1}=3, N_{2}=$ $M_{2}=4$, and $N=M=7$. On the other hand, we have to set $N_{1}^{\prime}=M_{1}^{\prime}=3, N_{2}^{\prime}=M_{2}^{\prime}=5$, and $N^{\prime}=M^{\prime}=$ 10 to reach the DOFs goal by use of the coprime-STAP. Obviously, the O2LN-STAP can achieve the DOFs aim with fewer physical array elements and transmitting pulses than the coprime-STAP. In addition, as is reported in the literature 


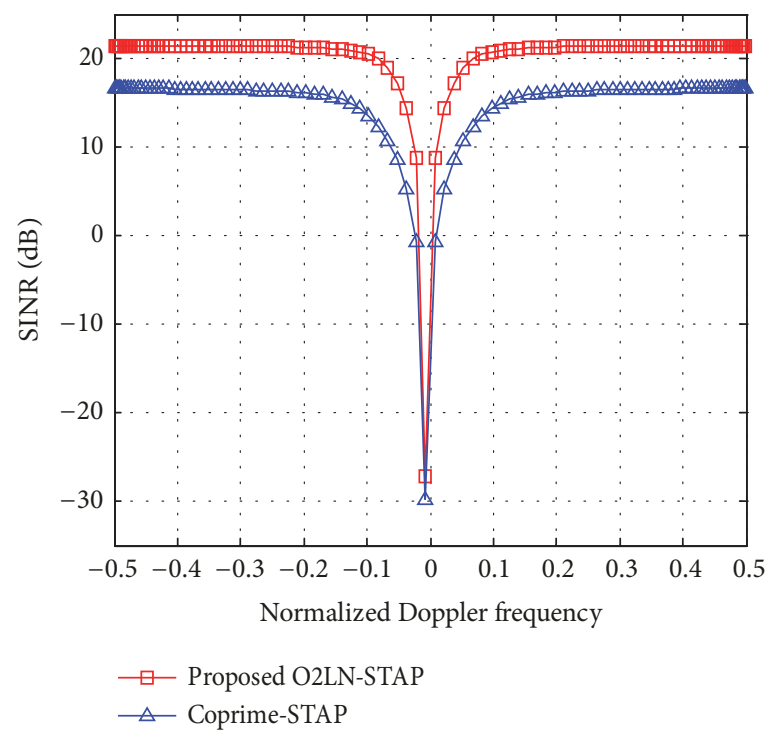

FIGURE 9: Optimum output SINR performances versus target normalized Doppler frequency with $\mathrm{N}=6$ array elements and $\mathrm{M}=6$ transmitting pulses.

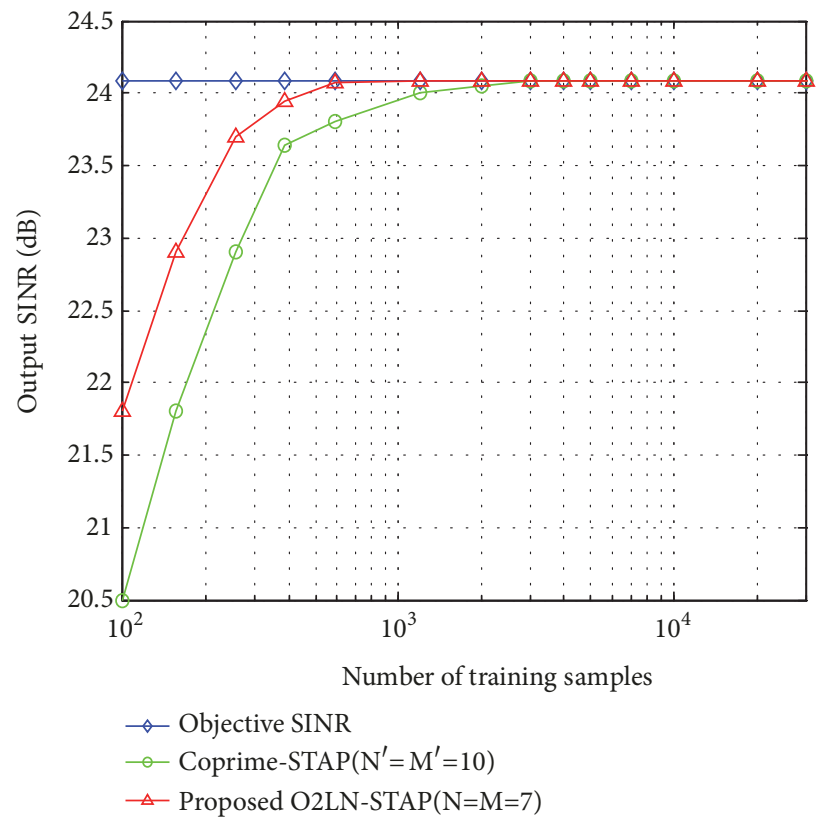

FIGURE 10: Output SINR performances versus the number of training samples.

$[30,32]$, the coprime-STAP method is also derived from the traditional CNCM $\mathbf{R}_{u}$. Hence, there also exists SINR loss due to the estimation error when the true traditional $\mathrm{CNCM}$ is replaced by its estimation in practice. Thus, based on the CNCM estimation, we still further analyze the output SINR performance versus the number of training samples for the O2LN-STAP and the coprime-STAP. Considering that the PC technique is more suitable for the sample-starved environment than the SMI algorithm as aforementioned before, we only use the $\mathrm{PC}$ technique to implement the practical STAP filter for both O2LN-STAP and coprime-STAP in the comparative simulation in this subsection. The results are presented in Figure 10. The objective curve in the figure is indeed the theoretical upper bound of the output SINR corresponding to the V-ULA-UPRI-16x16 STAP system. We can see that the O2LN-STAP and the coprime-STAP can converge to the objective SINR level with different number of training samples. Concretely, the O2LN-STAP exhibits faster convergence property and higher SINR in the case of a small number of training samples than the coprime-STAP. This is because that the traditional CNCM for the O2LN-STAP has smaller size than that for the coprime-STAP in the conditions of $N=M=7$ and $N^{\prime}=M^{\prime}=10$, which makes the requirement of training samples for estimating the small-size 
CNCM less than that for estimating the large-size CNCM, according to the RMB rule. Therefore, in contrast with the coprime-STAP, the O2LN-STAP is more potential for clutter suppression with fewer training samples, physical array elements, and pulses in the sample-limited heterogeneous environment.

Finally, note that the key point of our research is focused on how to enhance the DOFs based on the nested space-time structure and illustrating the advantages of this structure. All the comparative simulation results are carried out by combining different space-time structures with common STAP procedure including CCM estimation and angleDoppler filter. Then, the computational complexity is mainly embedded in the STAP processing related to CCM estimation or angle-Doppler filter, but neither in the nested space-time structure nor in other contrastive structures. Hence, by analyzing the computational complexity of the algorithms with respect to CCM estimation and angle-Doppler filter, one can further discuss the computational complexity of the STAP methods concerning the different space-time structures used herein.

\section{Conclusion}

In this paper, we have considered the issue of STAP with nested array and nested PRI for clutter suppression on the airborne/spaceborne MTI radar and proposed an O2LNSTAP strategy. The O2LN-STAP is capable of providing enhanced available DOFs in the difference coarray and copulses domains to further improve the STAP performance. Simulation results have validated the theoretical derivations of the proposed O2LN-STAP and revealed the following. (1) Under the constraint of the same number of physical array elements and transmitting pulses, the O2LN-STAP can offer higher available DOFs than the traditional uniform-STAP and the coprime-STAP, which makes an improved angleDoppler resolution, lower side-lobe levels, higher SINR, and better MDV performance. (2) For achieving a specified number of DOFs to improve the STAP performance, the O2LN-STAP needs fewer number of physical array elements and transmitting pulses than the traditional uniform-STAP and the coprime-STAP, which reduces the hardware complexity and power consumption. (3) The O2LN-STAP is more potential for clutter suppression with fewer training samples, physical array elements, and pulses in the samplelimited heterogeneous environment than the coprime-STAP. In addition, in contrast with the coprime-STAP, the enhanced available DOFs and the improved performance of the O2LNSTAP are obtained at the sacrifice of array mutual coupling performance. Hence, the issue for reducing the mutual coupling while holding the DOFs advantage of the nested class of STAP method might be an interesting research work in the future.

\section{Appendix}

Proof of the Proposition 3. Firstly, fix the total number of physical array elements and transmitting pulses to $N$ and
$M$, respectively. $N$ and $M$ are both positive integers. For the O2LN-STAP, we have

$$
\begin{aligned}
& N=N_{1}+N_{2}, \\
& M=M_{1}+M_{2} .
\end{aligned}
$$

Using the inequality of arithmetic mean and geometric mean (AM-GM inequality), we can get that

$$
N_{2}\left(N_{1}+1\right) \leq \frac{N_{2}^{2}+\left(N_{1}+1\right)^{2}}{2},
$$

with equality if (and only if) $N_{2}=\left(N_{1}+1\right)$. Combining (A.1) and the constraint that $N_{1}$ and $N_{2}$ should be both positive integers, we can further obtain the maximum value of the term $N_{2}\left(N_{1}+1\right)$, which can be written as

$$
\begin{aligned}
& \text { for odd } N, A_{1}=\max \left\{N_{2}\left(N_{1}+1\right)\right\}=\frac{(N+1)^{2}}{4}, \\
& \text { for even } N, B_{1}=\max \left\{N_{2}\left(N_{1}+1\right)\right\}=\frac{N^{2}}{4}+\frac{N}{2},
\end{aligned}
$$

where $N_{1}$ and $N_{2}$ are computed by

for odd $N$,

$$
\begin{aligned}
& N_{1}=\frac{N-1}{2}, \\
& N_{2}=\frac{N+1}{2},
\end{aligned}
$$

$$
\text { for even } N, N_{1}=N_{2}=\frac{N}{2} \text {. }
$$

Following a similar deducing process, we can also obtain the maximum value of the term $M_{2}\left(M_{1}+1\right)$ given by

$$
\begin{aligned}
\text { for odd } M, A_{2} & =\max \left\{M_{2}\left(M_{1}+1\right)\right\} \\
& =\frac{(M+1)^{2}}{4}, \\
\text { for even } M, B_{2} & =\max \left\{M_{2}\left(M_{1}+1\right)\right\} \\
& =\frac{M^{2}}{4}+\frac{M}{2},
\end{aligned}
$$

where $M_{1}$ and $M_{2}$ are given by

for odd $M$,

$$
\begin{aligned}
& M_{1}=\frac{M-1}{2}, \\
& M_{2}=\frac{M+1}{2},
\end{aligned}
$$

for even $M, M_{1}=M_{2}=\frac{M}{2}$.

On the other hand, for the coprime-STAP, we have

$$
\begin{aligned}
& N=N^{\prime}=2 N_{1}^{\prime}+N_{2}^{\prime}-1, \\
& M=M^{\prime}=2 M_{1}^{\prime}+M_{2}^{\prime}-1 .
\end{aligned}
$$




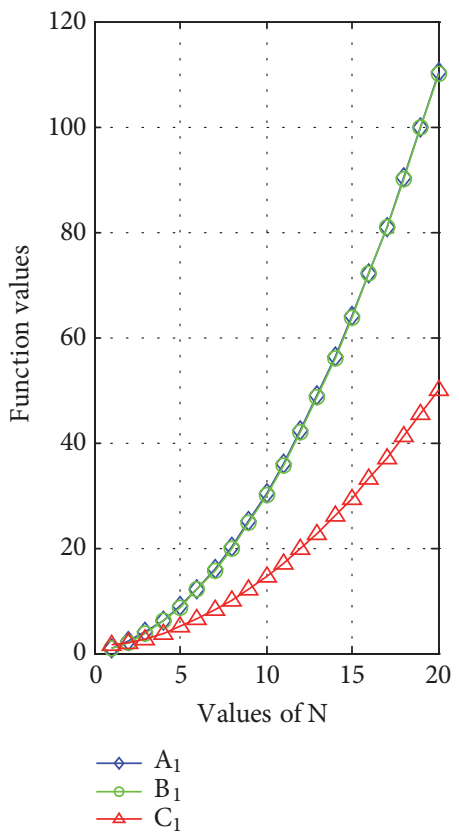

(a)

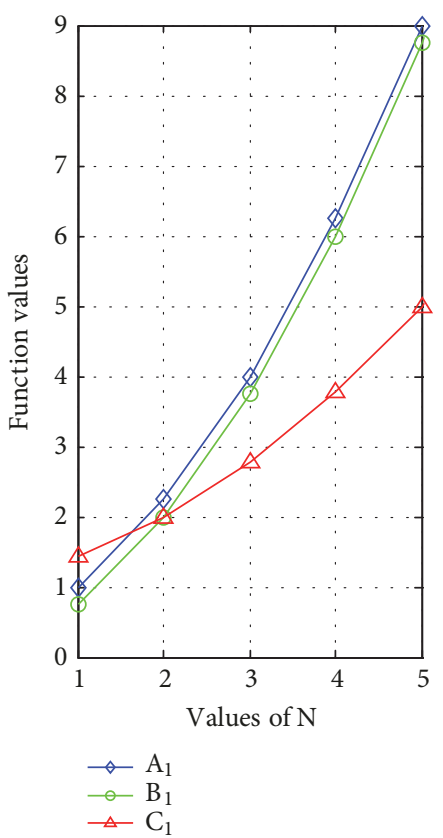

(b)

FiguRE 11: Three different function values versus N. (a) Original curves. (b) Close-up of the original curves.

Still using the AM-GM inequality, we can get that

$$
N_{1}^{\prime} N_{2}^{\prime}+1 \leq \frac{N_{1}^{\prime 2}+N_{2}^{\prime 2}}{2}+1
$$

with equality if (and only if) $N_{1}^{\prime}=N_{2}^{\prime}$. Now if we do not consider the constraint that $N_{1}^{\prime}$ and $N_{2}^{\prime}$ should be both integers, we can get the maximum value of $N_{1}^{\prime} N_{2}^{\prime}+1$ according to (A.7) and (A.8), which is expressed as

$$
C_{1}=\max \left\{N_{1}^{\prime} N_{2}^{\prime}+1\right\}=\frac{(N+1)^{2}}{9}+1,
$$

where $N_{1}^{\prime}$ and $N_{2}^{\prime}$ are given by

$$
N_{1}^{\prime}=N_{2}^{\prime}=\frac{N+1}{3}
$$

Similarly, we can get the maximum values of $M_{1}^{\prime} M_{2}^{\prime}+1$, which is written by

$$
C_{2}=\max \left\{M_{1}^{\prime} M_{2}^{\prime}+1\right\}=\frac{(M+1)^{2}}{9}+1,
$$

where $M_{1}^{\prime}$ and $M_{2}^{\prime}$ are given by

$$
M_{1}^{\prime}=M_{2}^{\prime}=\frac{M+1}{3}
$$

Note that $A_{1}, B_{1}$, and $C_{1}$ can be seen as three different function values with respect to the variable $N$. And their function values versus the variable $N$ are presented in Figure 11. Figure 11(b) is the close-up of Figure 11(a). It is clear that the values of $A_{1}$ and $B_{1}$ are both larger than $C_{1}$ when $2<$
$N \leq 20$, and $B_{1}$ is equivalent to $C_{1}$ when $N=2$. Additionally, it is noted that the second-order derivatives of $A_{1}, B_{1}$, and $C_{1}$ with respect to $N$ are $1 / 2,1 / 2$, and $2 / 9$, respectively. That is to say, since the function values of $A_{1}$ and $B_{1}$ increase more rapidly with the increasing of $N$ than that of $C_{1}$, the curve $C_{1}$ would no longer intersect the curves $A_{1}$ and $B_{1}$ after $N=20$. It means that the values of $A_{1}$ and $B_{1}$ are always larger than that of $C_{1}$ when $N>2$, which is not shown in Figure 11 due to the space limitation. Similarly, we can also say that the values of $A_{2}$ and $B_{2}$ are always larger than that of $C_{2}$ when $M>2$.

Furthermore, we rewrite the expression of $D_{2-\text { nested }}^{\text {opt }}$ and $D_{\text {coprime }}$ as follows:

$$
\begin{aligned}
& D_{2-\text { nested }}^{\text {opt }}= \begin{cases}A_{1} A_{2} & N: \text { odd } ; M: \text { odd } ;(a) \\
B_{1} B_{2} & N: \text { even } ; M: \text { even } ;(b) \\
A_{1} B_{2} & N: \text { odd } ; M: \text { even } ;(c) \\
B_{1} A_{2} & N: \text { even } ; M: \text { odd } ;(d),\end{cases} \\
& D_{\text {coprime }}<C_{1} C_{2} .
\end{aligned}
$$

Note that if we consider the constraint that $N_{1}^{\prime}$ (or $M_{1}^{\prime}$ ) and $N_{2}^{\prime}$ (or $M_{2}^{\prime}$ ) are coprime integers and $N_{1}^{\prime}<N_{2}^{\prime}\left(\right.$ or $\left.M_{1}^{\prime}<M_{2}^{\prime}\right)$, the conditions of (A.10) and (A.12) cannot be satisfied and the maximum values of $C_{1}$ and $C_{2}$ cannot be even reached too, which results in expression (A.14). Therefore, considering the aforementioned analysis results that $A_{1}>C_{1}, B_{1}>C_{1}$ when $N>2$ and $A_{2}>C_{2}, B_{2}>C_{2}$ when $M>2$, we can deduce the conclusion that $D_{2-\text { nested }}^{\text {opt }}>D_{\text {coprime }}$ when $N>2$ and $M>2$ according to (A.13) and (A.14). Thus, Proposition 3 has been proved. 


\section{Data Availability}

The datasets supporting the conclusions of this article are generated using MATLAB software by authors according to the radar parameters described in the manuscript.

\section{Conflicts of Interest}

The authors declare that they have no competing interests.

\section{Acknowledgments}

The authors express their gratitude to the editor and anonymous reviewers for their helpful comments and suggestions that improved this paper.

\section{References}

[1] W. L. Melvin, "A STAP overview," IEEE Aerospace and Electronic Systems Magazine, vol. 19, no. 1, pp. 19-35, 2004.

[2] R. Klemm, Principles of Space-Time Adaptive Processing, The Institution of Engineering and Technology, London, UK, 2006.

[3] J. R. Guerci, Space-Time Adaptive Processing for Radar, Artech House, Boston, Mass, USA, 2003.

[4] C. Chen and P. P. Vaidyanathan, "MIMO radar space-time adaptive processing using prolate spheroidal wave functions," IEEE Transactions on Signal Processing, vol. 56, no. 2, pp. 623635, 2008.

[5] Z. Liu, X. Wei, and X. Li, "Aliasing-free moving target detection in random pulse repetition interval radar based on compressed sensing," IEEE Sensors Journal, vol. 13, no. 7, pp. 2523-2534, 2013.

[6] J. Ward, "Space-time adaptive processing with sparse antenna arrays," in Proceedings of the 1998 32nd Asilomar Conference on Signals, Systems \& Computers. Part 1 (of 2), pp. 1537-1541, November 1998.

[7] Z. Yang, G. Quan, Y. Ma, and J. Huang, "Compressive spacetime adaptive processing airborne radar with random pulse repetition interval and random arrays," in Proceedings of the 2016 4th International Workshop on Compressed Sensing Theory and its Applications to Radar, Sonar and Remote Sensing (CoSeRa), pp. 247-251, Aachen, Germany, September 2016.

[8] D. Cohen, A. Dikopoltsev, and Y. C. Eldar, "Extensions of subNyquist radar: reduced time-on-target and cognitive radar," in Proceedings of the 3rd Int. Workshop CoSeRa, vol. 62, pp. 31-35, 2015.

[9] A. Sarajedini, "Adaptive array thinning for space-Time beamforming," in Proceedings of the 33rd Asilomar Conference on Signals, Systems, and Computers, ACSSC 1999, pp. 1572-1576, USA, October 1999.

[10] A. Moffet, "Minimum-redundancy linear arrays," IEEE Transactions on Antennas and Propagation, vol. 16, no. 2, pp. 172-175, 1968.

[11] S. U. Pillai, Y. B. Ness, and F. Haber, "A new approach to array geometry for improved spatial spectrum estimation," Proceedings of the IEEE, vol. 73, no. 10, pp. 1522-1524, 1985.

[12] S. U. Pillai and F. Haber, "Statistical analysis of a high resolution spatial spectrum estimator utilizing an augmented covariance matrix," Institute of Electrical and Electronics Engineers Transactions on Acoustics, Speech and Signal Processing, vol. 35, no. 11, pp. 1517-1523, 1987.
[13] Y. I. Abramovich, D. A. Gray, A. . Gorokhov, and N. K. Spencer, "Positive-definite Toeplitz completion in DOA estimation for nonuniform linear antenna arrays. I. Fully augmentable arrays," IEEE Transactions on Signal Processing, vol. 46, no. 9, pp. 24582471, 1998.

[14] Y. I. Abramovich, N. K. Spencer, and A. Y. Gorokhov, "Positivedefinite Toeplitz completion in DOA estimation for nonuniform linear antenna arrays. II. Partially augmentable arrays," IEEE Transactions on Signal Processing, vol. 47, no. 6, pp. 15021521, 1999.

[15] D. H. Johnson and D. E. Dudgeon, Array Signal Processing Concepts and Techniques, Prentice-Hall, Englewood Cliffs, NJ, USA, 1993.

[16] C. S. Ruf, "Numerical annealing of low-redundancy linear arrays," IEEE Transactions on Antennas and Propagation, vol. 41, no. 1, pp. 85-90, 1993.

[17] P. P. Vaidyanathan and P. Pal, "Sparse sensing with co-prime samplers and arrays," IEEE Transactions on Signal Processing, vol. 59, no. 2, pp. 573-586, 2011.

[18] P. Pal and P. P. Vaidyanathan, "Coprime sampling and the music algorithm," in Proceedings of the IEEE Digital Signal Processing Workshop and IEEE Signal Processing Education Workshop (DSP/SPE '11), pp. 289-294, IEEE, Sedona, Ariz, USA, January 2011.

[19] S. Qin, Y. D. Zhang, and M. G. Amin, "Generalized coprime array configurations for direction-of-arrival estimation," IEEE Transactions on Signal Processing, vol. 63, no. 6, pp. 1377-1390, 2015.

[20] G. Di Martino and A. Iodice, "Passive beamforming with coprime arrays," IET Radar, Sonar \& Navigation, vol. 11, no. 6, pp. 964-971, 2017.

[21] E. BouDaher, Y. Jia, F. Ahmad, and M. G. Amin, "Multifrequency co-prime arrays for high-resolution direction-ofarrival estimation," IEEE Transactions on Signal Processing, vol. 63, no. 14, pp. 3797-3808, 2015.

[22] P. Pal and P. P. Vaidyanathan, "Nested arrays: a novel approach to array processing with enhanced degrees of freedom," IEEE Transactions on Signal Processing, vol. 58, no. 8, pp. 4167-4181, 2010.

[23] P. Pal and P. P. Vaidyanathan, "Nested arrays in two dimensions, Part I: Geometrical considerations," IEEE Transactions on Signal Processing, vol. 60, no. 9, pp. 4694-4705, 2012.

[24] A. Ahmed, Y. D. Zhang, and B. Himed, "Effective nested array design for fourth-order cumulant-based DOA estimation," in Proceedings of the 2017 IEEE Radar Conference, RadarConf 2017, pp. 0998-1002, USA, May 2017.

[25] K. Han and A. Nehorai, "Nested array processing for distributed sources," IEEE Signal Processing Letters, vol. 21, no. 9, pp. 11111114, 2014.

[26] K. Han and A. Nehorai, "Wideband gaussian source processing using a linear nested array," IEEE Signal Processing Letters, vol. 20, no. 11, pp. 1110-1113, 2013.

[27] W. Zheng, X. Zhang, and J. Shi, "Sparse extension array geometry for DOA estimation with nested MIMO radar," IEEE Access, vol. 5, pp. 9580-9586, 2017.

[28] X. Lan, Y. Li, and E. Wang, "A rare algorithm for 2D DOA estimation based on nested array in massive MIMO system," IEEE Access, vol. 4, pp. 3806-3814, 2016.

[29] R. Li, Y. Wang, Z. He, J. Li, and G. Sun, "Minimum redundancy space-time adaptive processing utilizing reconstructed covariance matrix," in Proceedings of the 2017 IEEE Radar Conference, RadarConf 2017, pp. 0722-0726, USA, May 2017. 
[30] C.-L. Liu and P. P. Vaidyanathan, "Coprime arrays and samplers for space-time adaptive processing," in Proceedings of the 40th IEEE International Conference on Acoustics, Speech, and Signal Processing, ICASSP 2015, pp. 2364-2368, Australia, April 2014.

[31] Y. Ma, Z. Yang, J. Huang, J. Huang, and L. Kang, "Spacetime adaptive processing airborne radar with coprime pulse repetition interval," in Proceedings of the 2016 CIE International Conference on Radar, RADAR 2016, pp. 1-4, China, October 2016.

[32] X. Wang, Z. Yang, H. Huang, J. Huang, and M. Jiang, "Spacetime adaptive processing for airborne radars with space-time coprime sampling structure," IEEE Access, vol. 6, pp. 2003120046, 2018.

[33] P. Vouras, "Fully Adaptive Space-Time Processing on nested arrays," in Proceedings of the 2015 IEEE International Radar Conference (RadarCon), pp. 0858-0863, Arlington, VA, USA, May 2015.

[34] P. Pal and P. P. Vaidyanathan, "Nested arrays in two dimensions, part II: Application in two dimensional array processing," IEEE Transactions on Signal Processing, vol. 60, no. 9, pp. 4706-4718, 2012. 


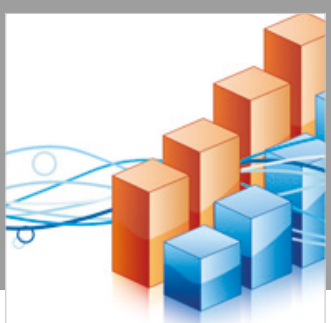

Advances in

Operations Research

\section{-n-m}
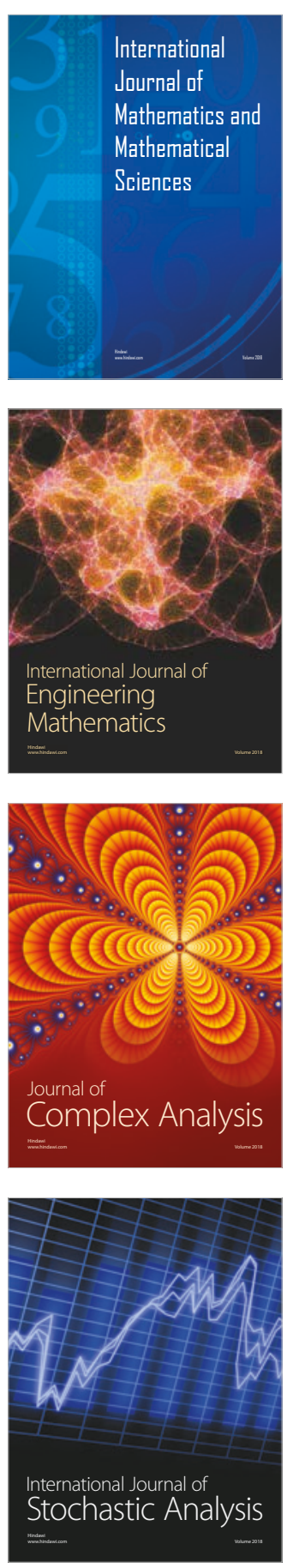
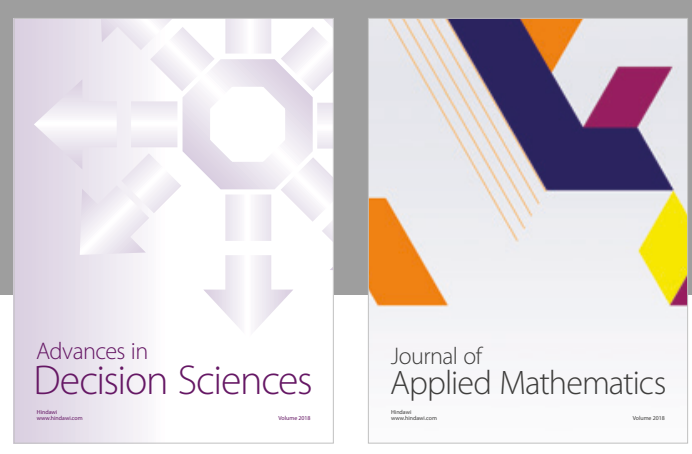

Journal of

Applied Mathematics
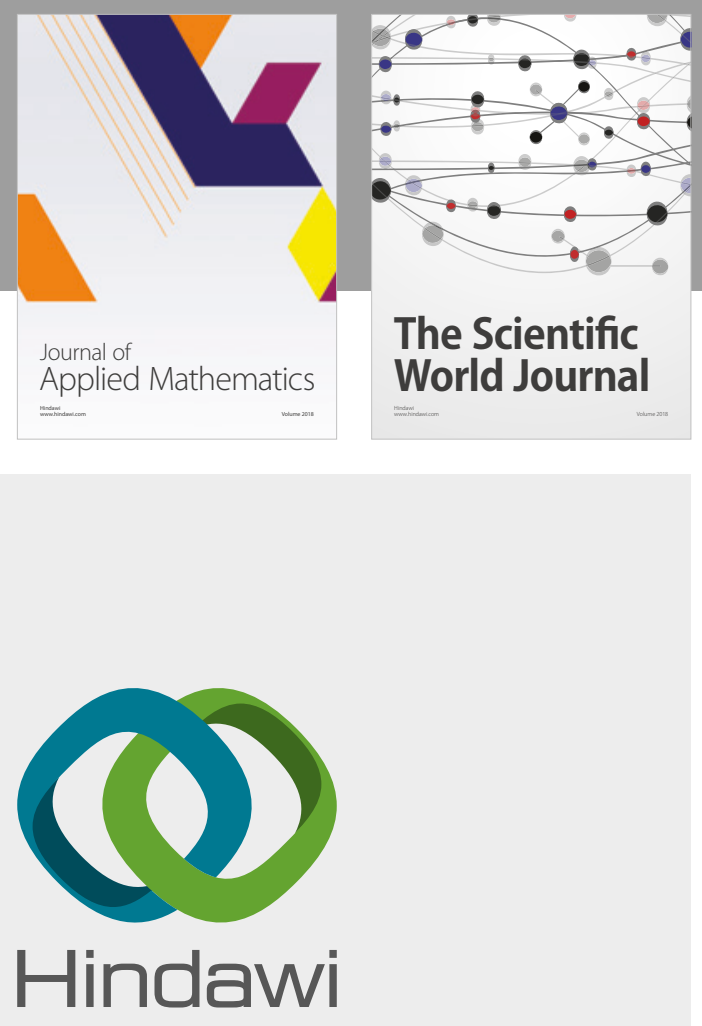

Submit your manuscripts at

www.hindawi.com

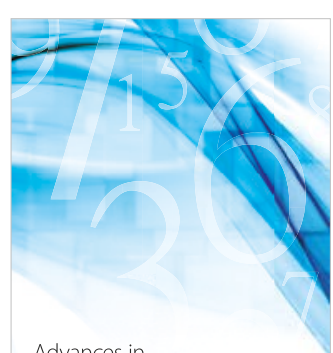

Advances in
Numerical Analysis
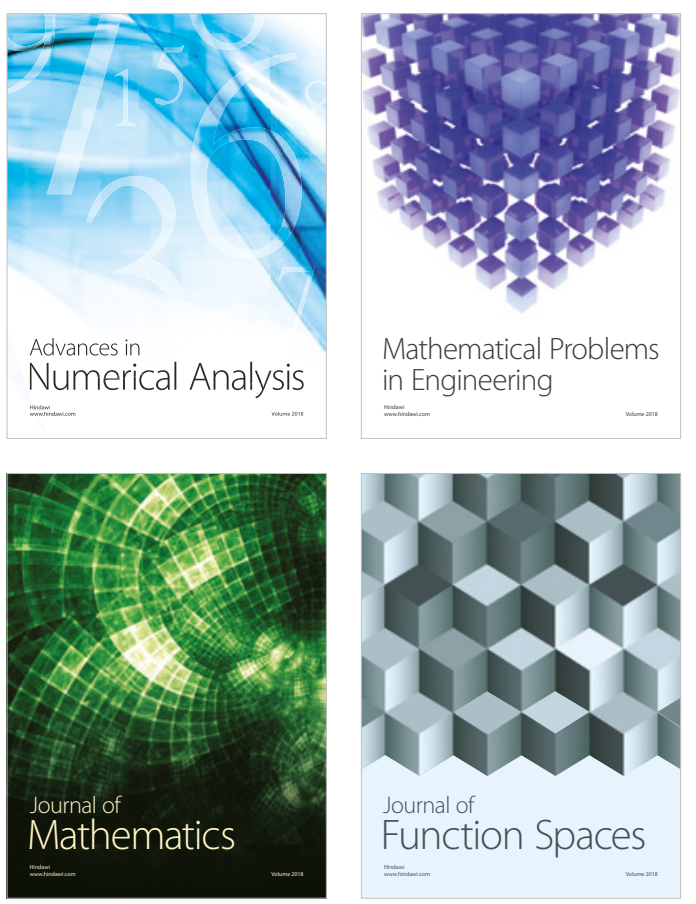

Mathematical Problems in Engineering

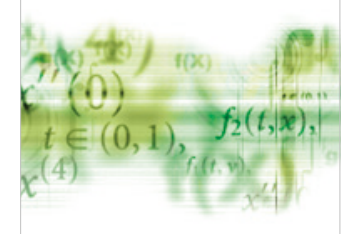

International Journal of

Differential Equations

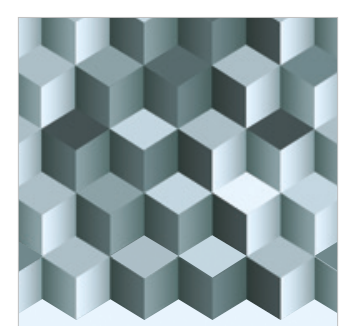

Journal of

Function Spaces

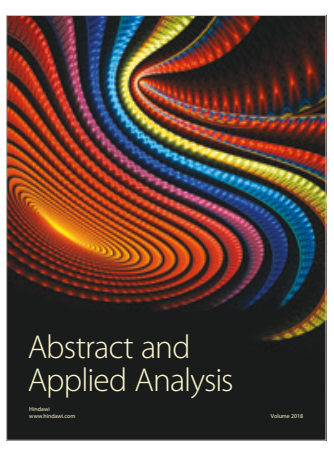

The Scientific

World Journal

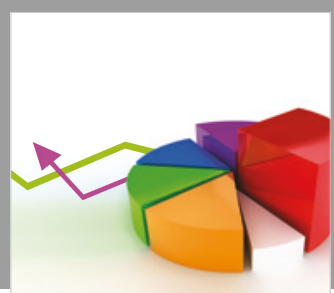

Journal of

Probability and Statistics
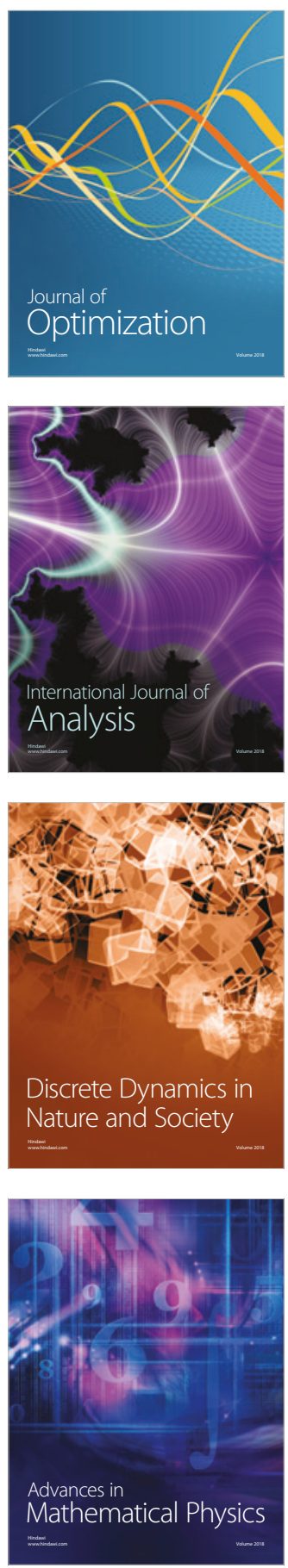